\title{
CAR T cells with dual targeting of CD19 and CD22 in adult patients with recurrent or refractory $B$ cell malignancies: a phase 1 trial
}

\author{
Jay Y. Spiegel (1,13, Shabnum Patel ${ }^{2,13}$, Lori Muffly ${ }^{102,13}$, Nasheed M. Hossain ${ }^{3}{ }^{3}$, Jean Oak ${ }^{4}$, \\ John H. Baird®1, Matthew J. Frank', Parveen Shiraz1, Bita Sahaf², Juliana Craig ${ }^{\circledR 1}$, Maria Iglesias', \\ Sheren Younes ${ }^{4}$, Yasodha Natkunam ${ }^{4}{ }^{4}$, Michael G. Ozawa ${ }^{4}$, Eric Yang ${ }^{4}$, John Tamaresis ${ }^{5}$, \\ Harshini Chinnasamy ${ }^{2}$, Zach Ehlinger ${ }^{2}$, Warren Reynolds ${ }^{2}$, Rachel Lynn ${ }^{2,12}$, Maria Caterina Rotiroti ${ }^{6}$, \\ Nikolaos Gkitsas², Sally Arai', Laura Johnston', Robert Lowsky', Robbie G. Majzner (i), \\ Everett Meyer', Robert S. Negrin', Andrew R. Rezvani', Surbhi Sidana ${ }^{1}$, Judith Shizuru ${ }^{1}$, \\ Wen-Kai Weng', Chelsea Mullins7, Allison Jacob7, Ilan Kirsch7, Magali Bazzano ${ }^{8}$, Jing Zhou ${ }^{8}$, \\ Sean Mackay8, Scott J. Bornheimer ${ }^{9}$, Liora Schultz ${ }^{2,6,10}$, Sneha Ramakrishna ${ }^{2,6}$, Kara L. Davis ${ }^{2,6}$, \\ Katherine A. Kong ${ }^{2}$, Nirali N. Shah ${ }^{10}{ }^{10}$, Haiying Qin ${ }^{10}{ }^{10}$, Terry Fry ${ }^{10,11}$, Steven Feldman ${ }^{2,14}$, \\ Crystal L. Mackall ${ }^{2,6,14 \bowtie}$ and David B. Miklos $\mathbb{1}^{1,2,14 \bowtie}$
}

\begin{abstract}
Despite impressive progress, more than $50 \%$ of patients treated with CD19-targeting chimeric antigen receptor T cells (CAR19) experience progressive disease. Ten of 16 patients with large B cell lymphoma (LBCL) with progressive disease after CAR19 treatment had absent or low CD19. Lower surface CD19 density pretreatment was associated with progressive disease. To prevent relapse with CD19- ${ }^{-}$or CD19 ${ }^{10}$ disease, we tested a bispecific CAR targeting CD19 and/or CD22 (CD19-22.BB.z-CAR) in a phase I clinical trial (NCT03233854) of adults with relapsed/refractory B cell acute lymphoblastic leukemia (B-ALL) and LBCL. The primary end points were manufacturing feasibility and safety with a secondary efficacy end point. Primary end points were met; $97 \%$ of products met protocol-specified dose and no dose-limiting toxicities occurred during dose escalation. In B-ALL $(n=17), 100 \%$ of patients responded with $88 \%$ minimal residual disease-negative complete remission (CR); in LBCL $(n=21)$, $62 \%$ of patients responded with $29 \%$ CR. Relapses were CD19-/10 in $50 \%$ (5 out of 10 ) of patients with B-ALL and $29 \%$ (4 out of 14) of patients with $L B C L$ but were not associated with CD22-/10 disease. CD19/22-CAR products demonstrated reduced cytokine production when stimulated with CD22 versus CD19. Our results further implicate antigen loss as a major cause of CAR $T$ cell resistance, highlight the challenge of engineering multi-specific CAR T cells with equivalent potency across targets and identify cytokine production as an important quality indicator for CAR T cell potency.
\end{abstract}

mpressive antitumor effects of chimeric antigen receptor-modified

T cells $^{1-10}$ and natural killer (NK) cells ${ }^{11}$ targeting CD19 (CAR19) have driven a paradigm shift in the treatment of relapsed or chemotherapy-refractory (relapsed/refractory) B cell malignancies. However, most patients treated with CAR19 experience disease progression. Clinical factors such as preCAR disease burden and serum lactate dehydrogenase $(\mathrm{LDH})^{5,8}$ have been linked to response to CAR19 therapies. Disease progression associated with loss of cell surface CD19 has been reported in 30-95\% of relapses after CAR19 therapy in B-ALL ${ }^{12}$, through a variety of mechanisms including splice mutations and retained intracellular CD19 $9^{12-16}$.
Several reports have also demonstrated that effective CAR T cell responses require high target antigen expression density ${ }^{17-21}$; resistance associated with diminished antigen density has been shown after treatment with a monospecific CD22-CAR ${ }^{22}$ and B cell maturation antigen $\mathrm{CARs}^{23}$. While CD19 is expressed at variable levels in $\mathrm{LBCL}^{21}$, the role of emergence of CD19-1/o LBCL in CAR19 resistance has not been well studied ${ }^{24,25}$. Engineering next-generation therapeutics to overcome newly defined mechanisms of resistance is an important unmet goal.

$\mathrm{CD} 22$ is a sialic acid-binding adhesion molecule largely restricted to the $\mathrm{B}$ cell lineage and expressed on most B-lineage malignancies ${ }^{26-30}$.

'Division of Blood and Marrow Transplantation and Cellular Therapy, Stanford University School of Medicine, Stanford, CA, USA. ${ }^{2}$ Center for Cancer Cell Therapy, Stanford Cancer Institute, Stanford University School of Medicine, Stanford, CA, USA. ${ }^{3}$ Division of Hematology/Oncology, Loyola University Medical Center, Chicago, IL, USA. ${ }^{4}$ Department of Clinical Pathology, Stanford University School of Medicine, Stanford, CA, USA. ${ }^{5}$ Department of Biomedical Data Science, Stanford University School of Medicine, Stanford, CA, USA. ${ }^{6}$ Department of Pediatrics-Hematology/Oncology, Stanford University School of Medicine, Stanford, CA, USA. ${ }^{7}$ Adaptive Biotechnologies, Seattle, WA, USA. ${ }^{8}$ soPlexis, Brantford, CT, USA. ${ }^{9}$ BD Biosciences, San Jose, CA, USA. ${ }^{10}$ Pediatric Oncology Branch Center for Cancer Research, National Institutes of Health, Bethesda, MD, USA. "Department of PediatricsHematology/Oncology, University of Colorado Anschutz and Children's Hospital Colorado, Denver, CO, USA. ${ }^{2}$ Present address: Lyell Immunopharma, San Francisco, CA, USA. ${ }^{13}$ These authors contributed equally; Jay Y. Spiegel, Shabnum Patel, Lori Muffly. ${ }^{14}$ These authors jointly supervised this work: Steven Feldman, Crystal Mackall, David B. Miklos.凶e-mail: cmackall@stanford.edu; dmiklos@stanford.edu 
In a study of children and young adults with B-ALL enriched for patients with progressive disease after CD19-directed therapy, CD22 CAR T cells induced a 73\% CR rate with equal effectiveness in $\mathrm{CD}_{19}{ }^{+}$and $\mathrm{CD}_{19}{ }^{-} \mathrm{B}-\mathrm{ALL}^{22,31}$. However, relapse was associated with the emergence of CD22 $2^{\text {lo }} \mathrm{B}$-ALL, illustrating the limitation of sequential targeting in B-ALL. In a phase I trial of adults with relapsed/refractory LBCL and B-ALL, we tested a bispecific CAR T targeting CD19 and/or CD22 (CD19-22.BB.Z) ${ }^{22}$ and demonstrated the feasibility of manufacturing in a closed system, safety of bispecific CAR T cells and clinical activity in both B-ALL and LBCL. The observed relapses with $\mathrm{CD} 19^{-/ \mathrm{lo}}$ and maintained CD22 expression could be due to reduced potency of the bispecific receptor toward CD22 versus CD19.

\section{Results}

Axicabtagene ciloleucel resistance is associated with $\mathrm{CD}^{-} 9^{-}$and CD19 ${ }^{\text {Io }}$ LBCL. To assess whether resistance to CAR19 therapy in LBCL is associated with $\mathrm{CD} 19^{-/ 10}$ relapse, we used the semiquantitative immunohistochemistry (IHC) H-score to measure CD19 expression at baseline and at disease progression in patients treated with axicabtagene ciloleucel (axi-cel) at our institution. Our cohort comprised 44 consecutive patients treated with axi-cel with available pretreatment tissue biopsies for IHC. With a median follow-up of 21 months (95\% confidence interval (CI) 11-24), median progression-free survival (PFS) was 6.1 months (95\% CI 3.1-not estimable); 23 patients (52\%) experienced progression. Sixteen of 23 patients with progression had postprogression biopsies available. Before axi-cel, the median CD19 H-score was 285 (interquartile range $(\mathrm{IQR})=240-285$ ) (Fig. 1a and Supplementary Fig. 1). Using an H-score cutoff of 150 to indicate CD19 positivity, 39 (89\%) patients were $\mathrm{CD}_{19}{ }^{+}$pretherapy. $\mathrm{CD} 19$ positivity was not associated with durable response (Fisher's exact test $P=1$ ). In contrast, only 6 of 16 samples studied (37.5\%) were $\mathrm{CD} 19^{+}$at disease progression. Among patients with paired pre- and posttherapy H-scores, 9 of $15(60 \%)$ converted from $\mathrm{CD} 19^{+}$pretherapy to $\mathrm{CD}^{-} 9^{-}$at relapse (McNemar test $P=0.003$ ) (Fig. $1 b$ ). Some postprogression biopsies showed complete loss of CD19, while others demonstrated diminished CD19 expression (Fig. 1c). Additionally, treating the pretreatment $\mathrm{H}$-score as a continuous variable also demonstrated no difference between patients with durable disease control versus those who experienced disease progression ( $t$-test $P=0.32$ ). These data demonstrate that progression after axi-cel therapy for LBCL is associated with emergent $\mathrm{CD} 19^{-/ 10}$ disease in a high percentage of patients, but pretreatment semiquantitative IHC measurement of CD19 expression does not identify patients at risk of relapse.

To evaluate whether quantitative assessment of CD19 cell surface density might predict outcomes after axi-cel and attempt to define a threshold level of antigen expression associated with relapse, we used quantitative flow cytometry to measure CD19 site density on viable single-cell suspensions obtained by fine needle aspiration. Preaxi-cel LBCL demonstrated substantial intra- and inter- patient variability in median CD19 site density as illustrated in Fig. 1d, which shows LBCLs from two representative patients, both of whom were $\mathrm{CD} 19^{+}$by IHC H-score. Patient A116 had a median CD19 site density of 6,538 molecules per cell and experienced durable disease control; in contrast, patient A140 had a median CD19 site density of 952 molecules per cell and experienced disease progression at 3 months after initial CR. Median preaxi-cel CD19 site density $(n=15)$ using quantitative flow cytometry was 6,396 molecules per cell (IQR $=3647-8540)$ with a range of $952-46,805$ molecules per cell (Fig. 1e). Using a penalized logistic regression model, patients with lower pretherapy median CD19 site density had significantly increased risk of progression after axi-cel $(P=0.03)$, with a $50 \%$ risk of progression for patients with 2,934 CD19 molecules per cell. Therefore, we selected 3,000 molecules per cell as a cutoff to define CD19 positivity (Extended Data Fig. 1). In our cohort, 3 of 4 patients with LBCL expressing $\leq 3,000$ CD19 molecules per cell experienced progression while 1 of 11 patients with LBCL expressing $>3,000$ molecules per cell had disease progression. Of 8 patients studied after axi-cel progression, 4 demonstrated a CD19 site density $\leq 3,000$ molecules per cell (Fig. If and Extended Data Fig. 2). The H-score did not correlate well with antigen site density (Spearman $r=0.28, P=0.38$; Fig. $1 \mathrm{~g}$ and Extended Data Fig. 2). These data corroborate the emergence of CD19 ${ }^{-/ \mathrm{lo}} \mathrm{LBCL}$ as a major cause of resistance to CAR19 therapy, suggesting that the risk of relapse increases in LBCLs expressing a median of $\leq 3,000$ molecules per cell before therapy, raising the prospect that quantitative flow cytometry, but not IHC, may identify LBCL patients a priori at risk of CD19-/lo relapse after CAR19.

CAR construct and clinical trial design. Previous data demonstrated that CD19 loss is an important mechanism of CAR19 failure in B-ALL. The data presented in this study similarly implicate absent or decreased cell surface CD19 as a mechanism of resistance after CAR19 in LBCL, providing a rationale for dual antigen targeting in B-ALL and LBCL. As reported previously ${ }^{22,32}$, we generated a CD1922.BB.z-CAR comprising a single cistron encoding the anti-CD19 murine FMC63 single-chain variable fragment ( $\mathrm{scFv}$ ) and fully human anti-CD22 m971 scFv (aCD19 vH-aCD22 vL-linker-aCD22 vH-aCD19 vL), followed by human CD8 hinge and transmembrane domains, $4-1 \mathrm{BB}$ costimulation and $\mathrm{CD} 3 \zeta$ activation domains. The CD19-22.BB.z-CAR was encoded by a self-inactivating lentiviral vector under control of an murine stem cell virus internal promoter (Fig. 2a and Supplementary Fig. 2). We conducted a phase I clinical trial of CD19-22.BB.z-CAR in patients with relapsed/refractory B-ALL and LBCL, evaluating the feasibility of manufacture and safety of CD19-22.BB.z-CAR as the primary end points. Eligible patients had disease relapsed or refractory after two or more lines of therapy and had measurable disease that expressed CD19. Patients received conditioning chemotherapy followed by CAR T cell infusion at 2 dose levels: $1 \times 10^{6} \mathrm{CAR}^{+}$cells kg-1 (DL1) and $3 \times 10^{6} \mathrm{CAR}^{+}$cells kg ${ }^{-1}$ (DL2) using a $3+3$ dose escalation phase that enrolled LBCL and

Fig. 1 | IHC demonstrates CD19-/lo disease postaxi-cel and quantitative flow cytometry of LBCL preaxi-cel therapy is associated with disease progression. $\mathbf{a}$, Preaxi-cel $\mathrm{H}$-scores did not distinguish long-term responders and those with progression postaxi-cel $(P=0.32$ by $t$-test, $P=1$ Fisher's exact test). Waterfall plot of CD19 IHC H-scores preaxi-cel therapy ( $n=44$ patients). The $\mathrm{H}$-score was calculated by the percentage of positive tumor cells (0-100) x stain intensity (1-3). The dashed line denotes the $\mathrm{H}$-score of 150, which was used to define antigen positivity. ND, not detectable. $\mathbf{b}$, Paired CD19 $\mathrm{H}$-scores preaxi-cel and at progression show significant differences ( $P=0.003$ by Wilcoxon signed-rank test). Using an $\mathrm{H}$-score cutoff of 150 , and the observed rate of CD19-/lo progression (10 out of 16 patients), the estimated $95 \%$ binomial $\mathrm{Cl}$ (Wilson score) for CD19-/lo progression was 38-82\%. $\mathrm{N} / \mathrm{A}$, no data point. c, Representative patients with IHC demonstrating decreased CD19 expression at the time of progression (A75, relapse H-score =160; $\mathrm{A} 62$ relapse $\mathrm{H}$-score $=120 ; \mathrm{A} 30$, relapse $\mathrm{H}$-score $=100 ; \mathrm{A} 53$, relapse $\mathrm{H}$-score $=0$ ) $\mathbf{d}$, Preaxi-cel site density by quantitative flow cytometry in a patient with ongoing response (A116) compared with a patient who experienced progression (A140) e, Preaxi-cel median CD19 site density by quantitative flow cytometry organized from highest (dark blue) to lowest (white) in 15 patients. Patients with lower site density were more likely to experience disease progression after axi-cel ( $P=0.03$ by Firth logistic regression). Based on the fit model, 3,000 molecules per cell was defined as the cutoff for CD19 positivity. $\mathbf{f}$, Median site density at the time of axi-cel progression $(n=8)$. Four patients had a site density $<3,000$ molecules per cell. $\mathbf{g}$, The preaxi-cel $\mathrm{H}$-score did not correlate with antigen site density by quantitative flow cytometry $(n=12)($ Spearman $r=0.28, P=0.38)$. 
B-ALL in one cohort. While a maximal tolerated dose was not identified, a third dose level at $1 \times 10^{7}$ cells kg-1 was not pursued due to the efficacy seen at DL2 and concern regarding the possible toxicity of cell doses higher than $3 \times 10^{6} \mathrm{cells} \mathrm{kg}^{-1}$ seen in other clinical trials. Therefore, the two disease-specific expansion cohorts received the recommended phase 2 dose of $3 \times 10^{6} \mathrm{CAR} \mathrm{T}$ cells kg-1 (DL2).

Patient characteristics. Thirty-nine patients were enrolled in two cohorts (B-ALL, $n=17$, LBCL, $n=22$ ); 38 patients received the
CD19-22.BB.z-CAR infusion while one patient with LBCL died during lymphodepletion due to progressive disease and sepsis (Extended Data Fig. 3). The median age for the cohort with LBCL who received the infusion was 69 years (range 25-78) (Table 1); 15 had double expressor (expression of c-MYC and BCL2) LBCL, 3 had high-grade B cell lymphoma with translocation of c-MYC and BCL2 and/or BCL6 (double-hit) and 4 had previous autologous stem cell transplant. All patients with LBCL were CAR-naive. In the cohort with B-ALL, median age was 47 years (range 26-68); a

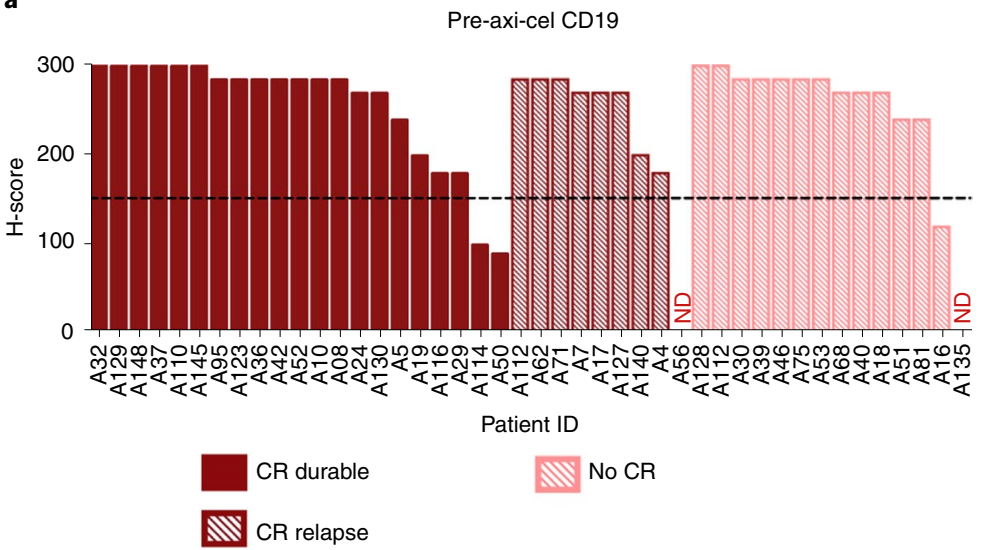

b

CD19 pre-axi-cel versus relapse

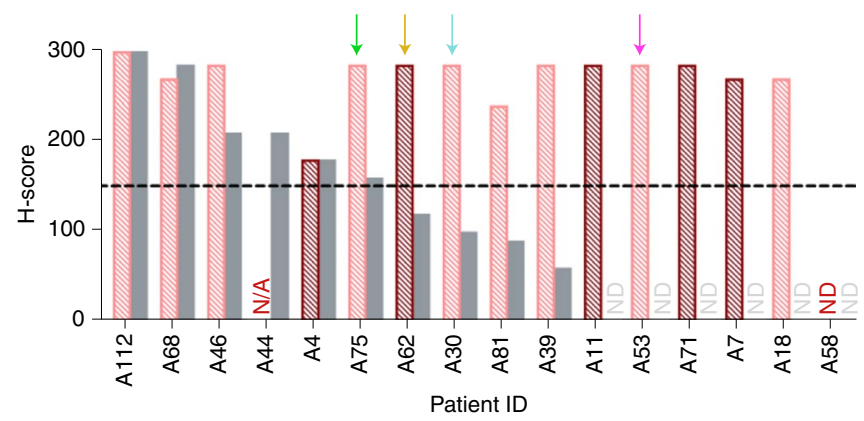

C A75 CD19 downregulation

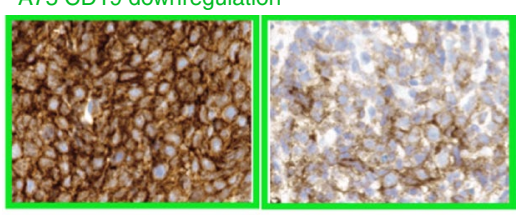

A62 CD19 downregulation

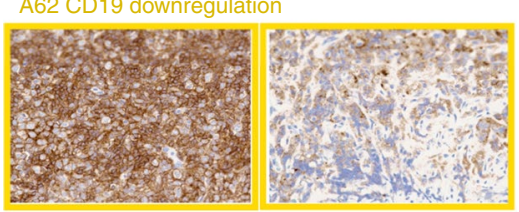

A30 CD19 downregulation

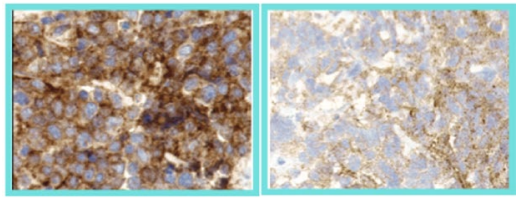

A53 CD19 loss

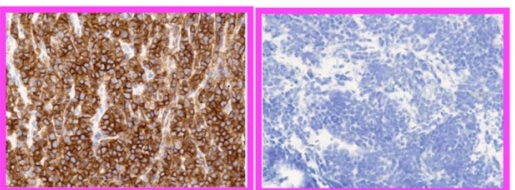

AN/N Pre-axi-cel

Post-progression

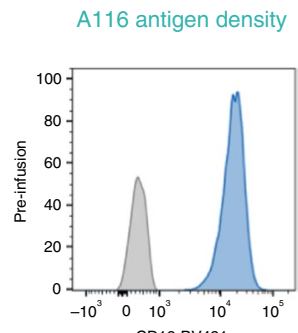

e

Pre-axi-cel

antigen density
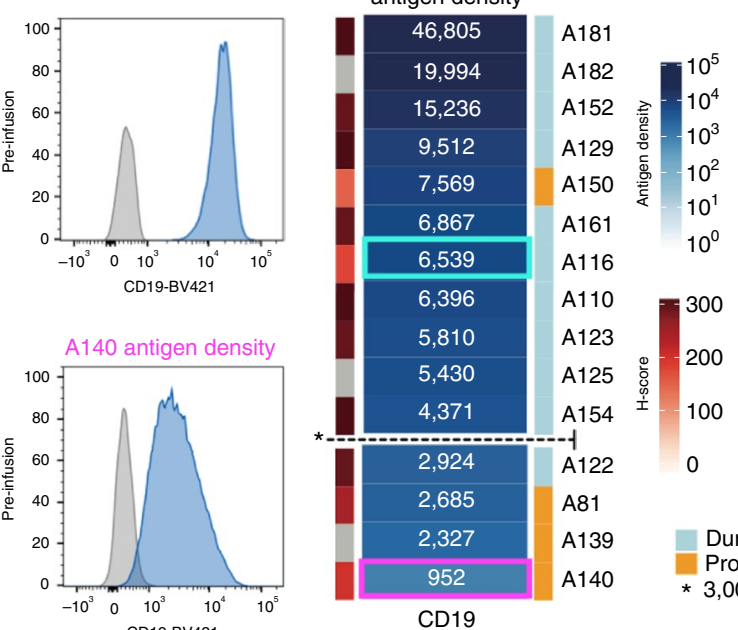

CD19-BV421

T cell control

Lymphoma f Antigen density $\quad$ g
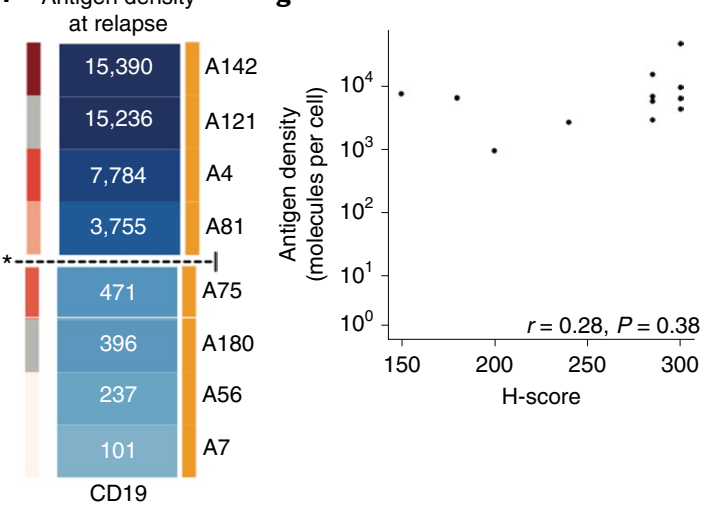
$71 \%$ had progressed after allogeneic hematopoietic cell transplantation (HCT), 65\% had previous CD19-directed therapy (including 1 patient receiving previous CAR T) and $29 \%$ had previous CD22-directed therapy (Table 2). Sixty-three percent had previous central nervous system (CNS) involvement and $12 \%$ had active CNS disease at the time of enrollment.

Feasibility of closed-system manufacturing. A primary objective of this clinical trial was to assess the feasibility of cell production using closed-system manufacturing in the CliniMACS Prodigy (Miltenyi Biotec), defined as $>80 \%$ of cell products meeting the protocol-specified cell dose. The manufacturing schema is illustrated in Fig. 2b and Extended Data Fig. 4a. Initially, a 7-9d manufacturing process (old matrix) utilized washout of the $\mathrm{T}$ cell activator (TransAct) on day 5. At DL1, 57\% (4 out of 7) of products met the prescribed cell dose within $7 \mathrm{~d}$ and $43 \%$ (3 out of 7 ) of products within $9 \mathrm{~d}$. To reduce manufacturing time while increasing dose level, we incorporated a process change to remove the TransAct on day 3 (new matrix), significantly shortening the culture time required to meet the dose, which is reflected by the increased total cell count by day $7(P<0.0001 ;$ Fig. $2 c)$ and decreased product doubling time $(P=0.04$; Fig. $2 \mathrm{~d})$. Among 39 patients who underwent apheresis, CAR T products meeting predefined release criteria were successfully manufactured in $100 \%$. Ninety-seven percent of products met the protocol-specified dose; $82 \%$ (32 out of 39 ) met the protocol-specified dose in $7 \mathrm{~d}$ ( 4 old matrix, 28 new matrix) (Extended Data Fig. 4b-d). Mean transduction efficiency was $60.1 \%$ (range 34.6-75.2\%; Fig. 2e), with an average vector copy number of 2.23 (range 1.31-4.0; Extended Data Fig. 4e).

Characterization of CD19-22.BB.z-CAR $\mathrm{T}$ infusion products. Samples were obtained from apheresis, postCD4/CD8 T cell enrichment and final CAR T product collection for cell subset analysis and to confirm enrichment in $\mathrm{T}$ cell populations and removal of leukemic cells. Compared to apheresis products, manufactured products demonstrated increased $\mathrm{T}$ cell and $\mathrm{CD}^{+}$populations, no change in $\mathrm{CD}^{+}$and natural killer $\mathrm{T}$ (NKT)-like subsets and depletion of $\mathrm{B}$ or leukemic cells (defined by $\mathrm{CD} 20^{+}$), NK cells, monocytes and neutrophils (Fig. 2f).

CD19-22.BB.z products showed a CD4 ${ }^{+}$predominance (Fig. $2 \mathrm{~g}$ ). To determine whether this was due to $\mathrm{T}$ cell enrichment postapheresis or the manufacturing process, we assessed the fold increase in $\mathrm{CD}^{+}$and $\mathrm{CD}^{+}$populations over the manufacturing process (Fig. 2h). While there was no difference in fold increase from apheresis to enrichment, there was a significant difference in fold increase between $\mathrm{CD}^{+}{ }^{+}$and $\mathrm{CD}^{+} \mathrm{T}$ cell subsets from enrichment to final product $(P<0.0001)$, implicating the manufacturing process in enriching the proportion of $\mathrm{CD}^{+}$cells in the manufactured product. Compared to enriched apheresis samples, products demonstrated enrichment in stem cell memory $\mathrm{T}\left(\mathrm{T}_{\mathrm{SCM}}\right)(P<0.0001)$ and central memory $\mathrm{T}\left(\mathrm{T}_{\mathrm{CM}}\right)(P<0.0001)$ cell subsets, no change in naive $\mathrm{T}\left(\mathrm{T}_{\mathrm{N}}\right)$ and effector memory $\mathrm{T}\left(\mathrm{T}_{\mathrm{EM}}\right)$ cell populations and a decrease in terminally differentiated effector memory $\left(\mathrm{T}_{\mathrm{EMRA}}\right)$ cell subsets $(P<0.0001$; Fig. 2i).

Toxicity. No dose-limiting toxicity (DLT) occurred during dose escalation and one DLT total occurred during the clinical trial. Cytokine release syndrome (CRS) of any grade occurred in 29 patients $(76 \%)$ with median onset $1 \mathrm{~d}$ after infusion (range 0-8) and lasted a median of $4 \mathrm{~d}$ (range 1-12) (Tables 1 and 2 and Extended Data Fig. 5). Grade $\geq 3$ CRS occurred in 2 patients (5\%). Neurological toxicity occurred in 14 patients (37\%; 9 with LBCL and 5 with B-ALL); 4 experienced $\geq$ grade 3 neurotoxicity. Onset of neurotoxicity occurred a median of $5 \mathrm{~d}$ after infusion (range 3-9) and lasted a median of $4 \mathrm{~d}$ (range 1-11). Both CRS and neurotoxicity were treated according to institutional guidelines, with 15 patients (39\%) receiving $\geq 1$ dose of tocilizumab (range 1-3) and $45 \%$ of patients receiving corticosteroids. All episodes of CRS and neurotoxicity resolved. Two patients had laboratory evidence of macrophage activation syndrome with hyperferritinemia and hypofibrinogenemia concurrent with grade $\geq 3$ neurotoxicity and received high-dose corticosteroids and anakinra ${ }^{31}$.

Response. The primary response assessment for LBCL was undertaken at three months after infusion. For patients with LBCL treated at the recommended phase II dose $(n=15)$, the overall response rate (ORR) and CR rate at 3 months were $40 \%$ (95\% CI 16-68\%) and $33 \%$ (95\% CI 12-62\%), respectively. For the entire patient cohort with LBCL $(n=21)$, the best ORR at any time point was $62 \%$ (95\% CI $38-82 \%$ ) and the CR rate was $29 \%$ (95\% CI $11-52 \%$ ). Five of 13 responders had improvement in response from month 1 to month 3 after infusion (Fig. 3a-c). With a median follow-up of 10 months (95\% CI 8.7-21.5), median overall survival was 22.5 months (95\% CI 8.3-not estimable; Fig. 3d), which could change with a longer follow-up. Median PFS was 3.2 months (95\% CI 1.25.5; Fig. 3e). To understand the kinetics of response in patients with LBCL treated with CD19-22.BB.z, we assessed change in lymphoma burden over time by cell-free circulating tumor DNA (ctDNA) in 16 patients with available diagnostic tumor samples (Extended Data Fig. 6) ${ }^{33}$. Four patients with ongoing clinical response had no detectable ctDNA at the time of the last assessment. Among 12 patients with disease progression, we observed an initial reduction in ctDNA that nadired 14-21 d postinfusion, with 9 patients demonstrating a rise in ctDNA at or before clinical progression. These findings suggest that progressive disease after CD19-22.BB.z-CAR in LBCL is associated with a robust early response followed by early acquired resistance.

Primary response for B-ALL was evaluated at 28 days postinfusion. All $(n=17)$ patients with B-ALL achieved response; 14 with $\mathrm{CR}(82 \%)$ and 3 with partial remission (Fig. 3f). One patient's

Fig. 2 | Characterization of CAR products throughout the manufacturing process reveals compositional and phenotypic changes. a, CD19-22-CD8. BB.z-CAR contained the CD19 FMC63 and CD22 M971 scFvs, CD8 $\alpha$ hinge and transmembrane domains, a 4-1BB costimulatory domain and a CD3 $\zeta$ domain. The unique bispecific structure shows FMC63 heavy chain proximal, followed by M971 light chain, M971 heavy chain and FMC63 light chain distal. b, CAR T manufacturing and clinical trial schema. The manufacturing schema shows the TransAct process change from old to new matrix. The clinical trial schema shows the screening, lymphodepletion, CAR T cell infusion and disease evaluation time points. LP, lumbar puncture. c, Improved culture expansion resulting from the new matrix manufacturing process compared to the old matrix $(P<0.0001$, two-tailed $t$-test $)$. $\mathbf{d}$, Significant reduction in doubling time with the new matrix process compared to the old matrix $(P=0.0411$, two-tailed $t$-test). e, No significant difference in transduction efficiency between old and new matrix ( $P=$ not significant (NS), two-tailed $t$-test). Overall average transduction efficiency was $60.1 \%$ ( $n=39$ individual products). $\mathbf{f}$, Composition of apheresis, CD4/8-enriched and CD19-22.BB.z product over time, looking at T cell (CD3 $\left.{ }^{+} C D 566^{-}\right), B$ cell or leukemic cell $\left(\mathrm{CD} 20^{+}\right), \mathrm{CD}^{+}, \mathrm{CD} 8^{+}, \mathrm{NKT}$-like $\left(\mathrm{CD} 3^{+} \mathrm{CD} 56^{+} \mathrm{CD} 16^{+}\right), \mathrm{NK}$, monocyte and neutrophil subsets. $\mathbf{g}$, Phenotyping of CAR T cell product reveals a skewing toward $\mathrm{CD} 4^{+}$cells $(n=39$ individual products). $\mathbf{h}$, Comparing the fold increase from apheresis to enrichment to final product reveals the skewing toward CD4 ${ }^{+}$cells that occurred during culture between enrichment and final product $(P<0.0001$, two-tailed $t$-test). $\mathbf{i}$, Phenotyping of T cell memory subsets revealed an enrichment in $\mathrm{T}_{\mathrm{SCM}}(P<0.0001$, two-tailed $t$-test $)$ and $\mathrm{T}_{\mathrm{CM}}(P<0.0001$, two-tailed $t$-test $)$ cell subsets and a depletion of the $T_{\mathrm{EMRA}}(P<0.0001$, two-tailed $t$-test) subset. There was no significant change in $T_{N}$ or $T_{E M}$ cell subsets between enrichment and CD19-22.BB.z product. 
response (SA8) improved to CR 6 months after infusion (Fig. 3g,h), leading to an overall CR rate of $88 \%$, all of whom were negative for minimal residual disease (MRD) at $10^{-4}$ bone marrow sensitivity
(Extended Data Fig. 7) or by positron emission tomography (PET)/ computed tomography (CT) for patients with extramedullary disease. After a median follow-up of 9.3 months (95\% CI 7.2-NE), a
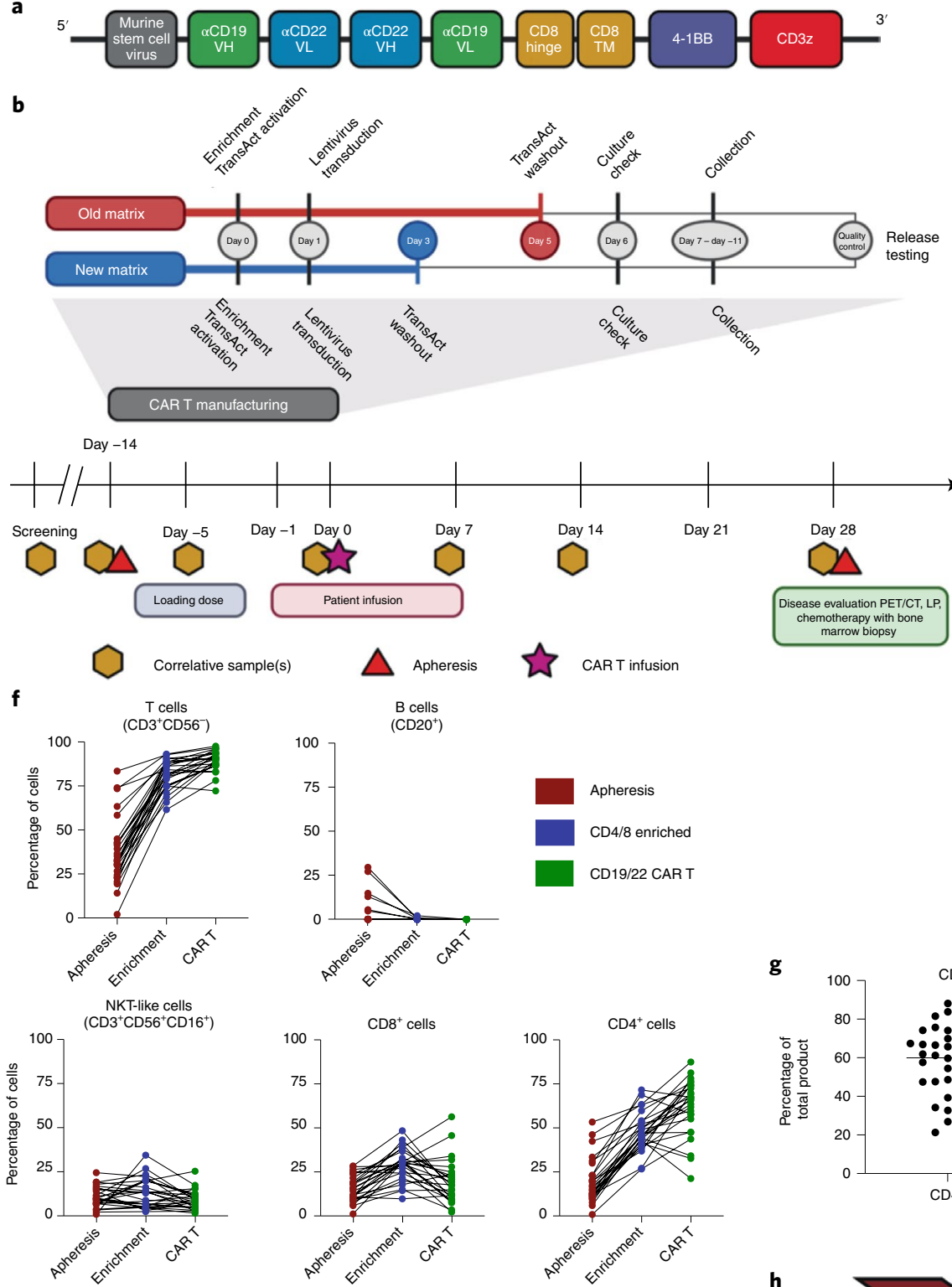

NK cells

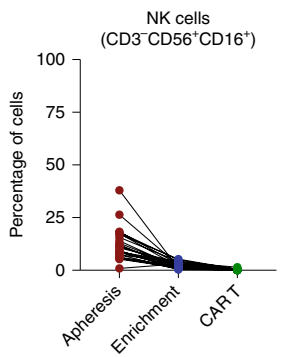

i
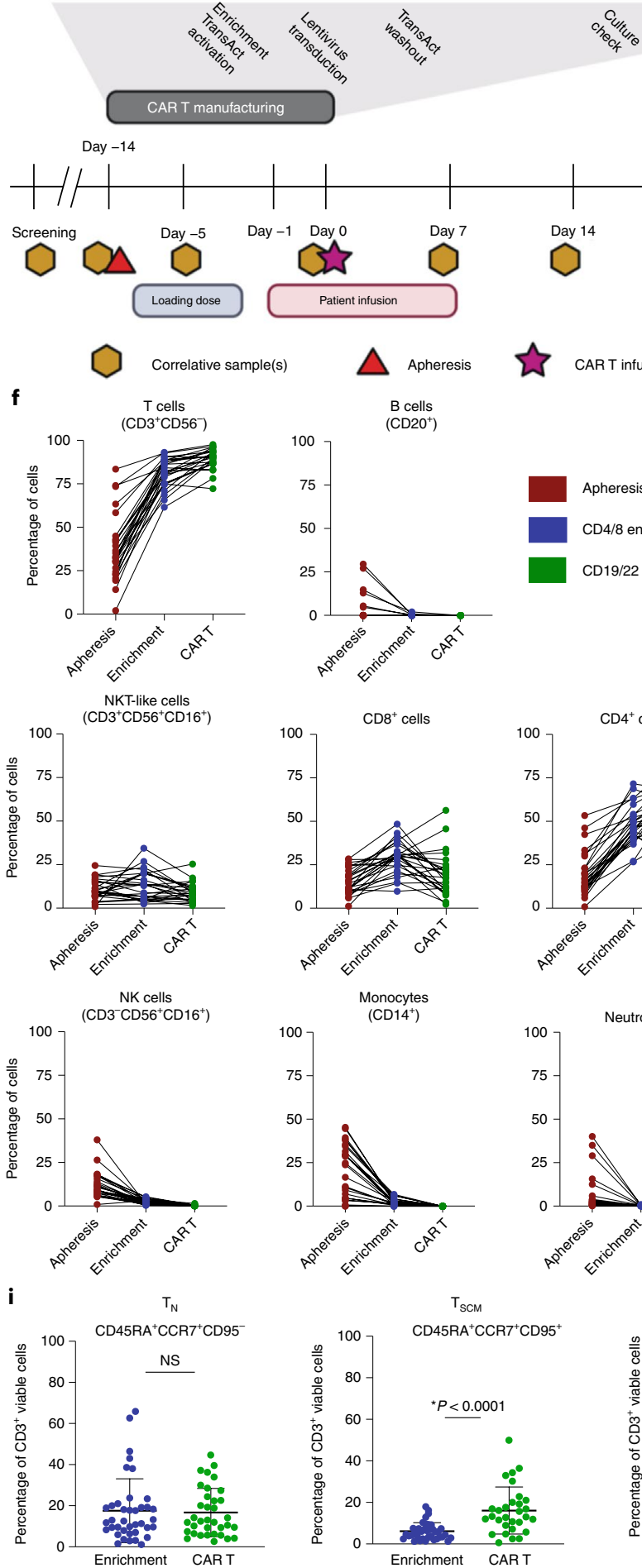

Day -1 Day 0
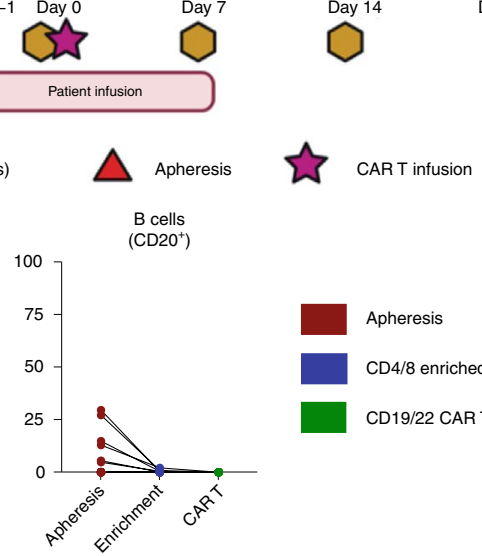

Apheresis

CD4/8 enriched

CD19/22 CAR T
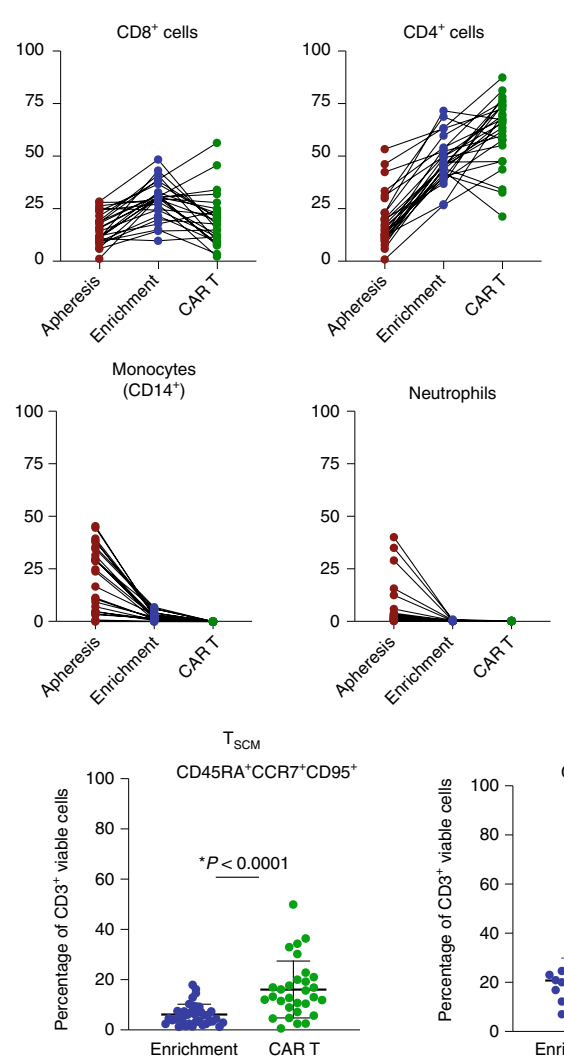
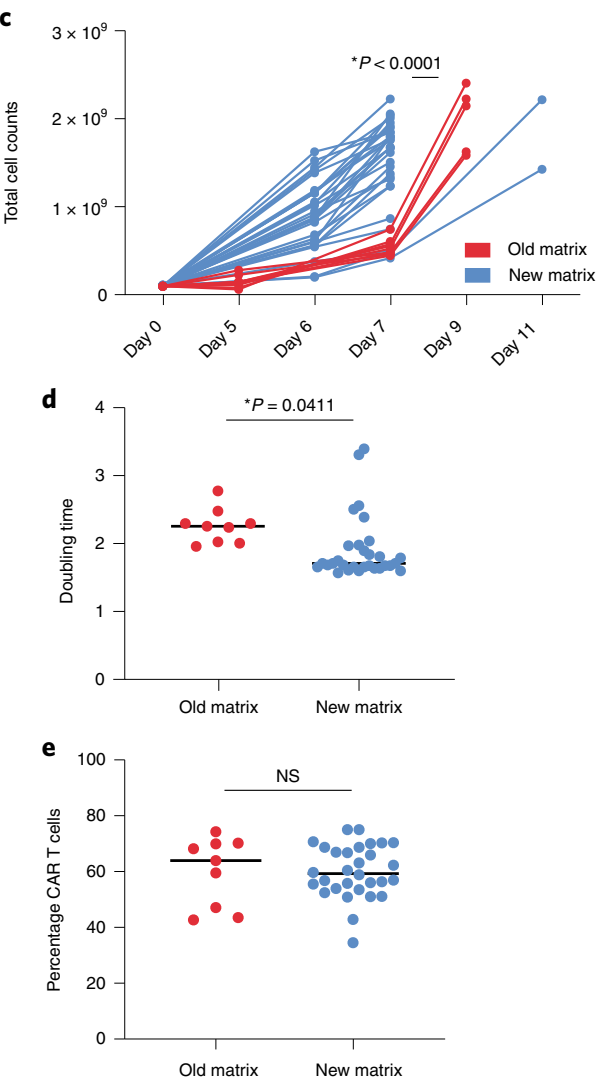

g

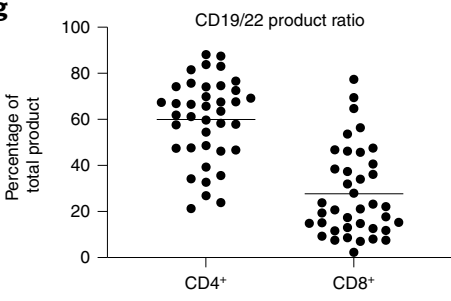

h

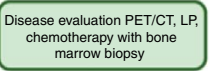
marrow biopsy
metherapy with bo,

h
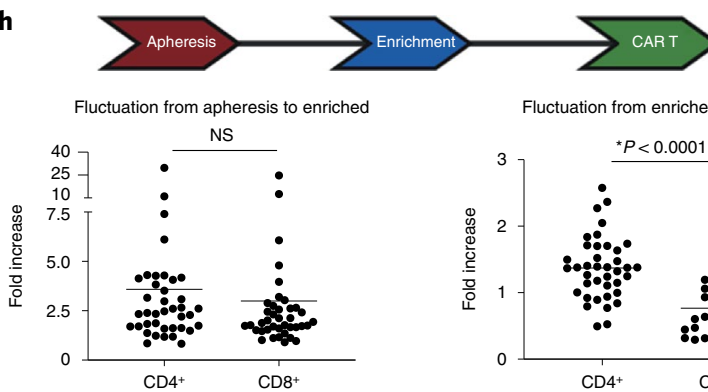

Fluctuation from enriched to product

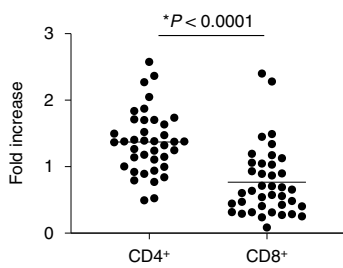

$T_{C M}$

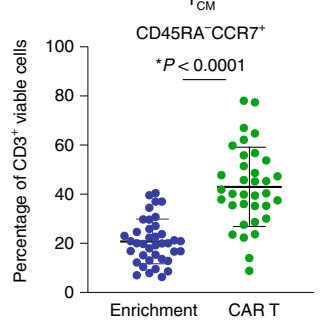

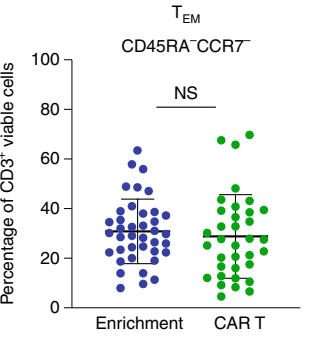

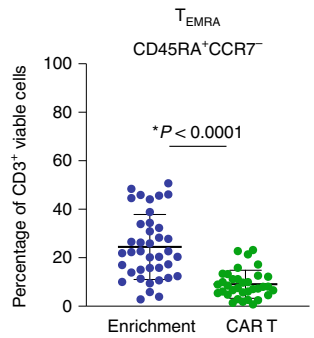




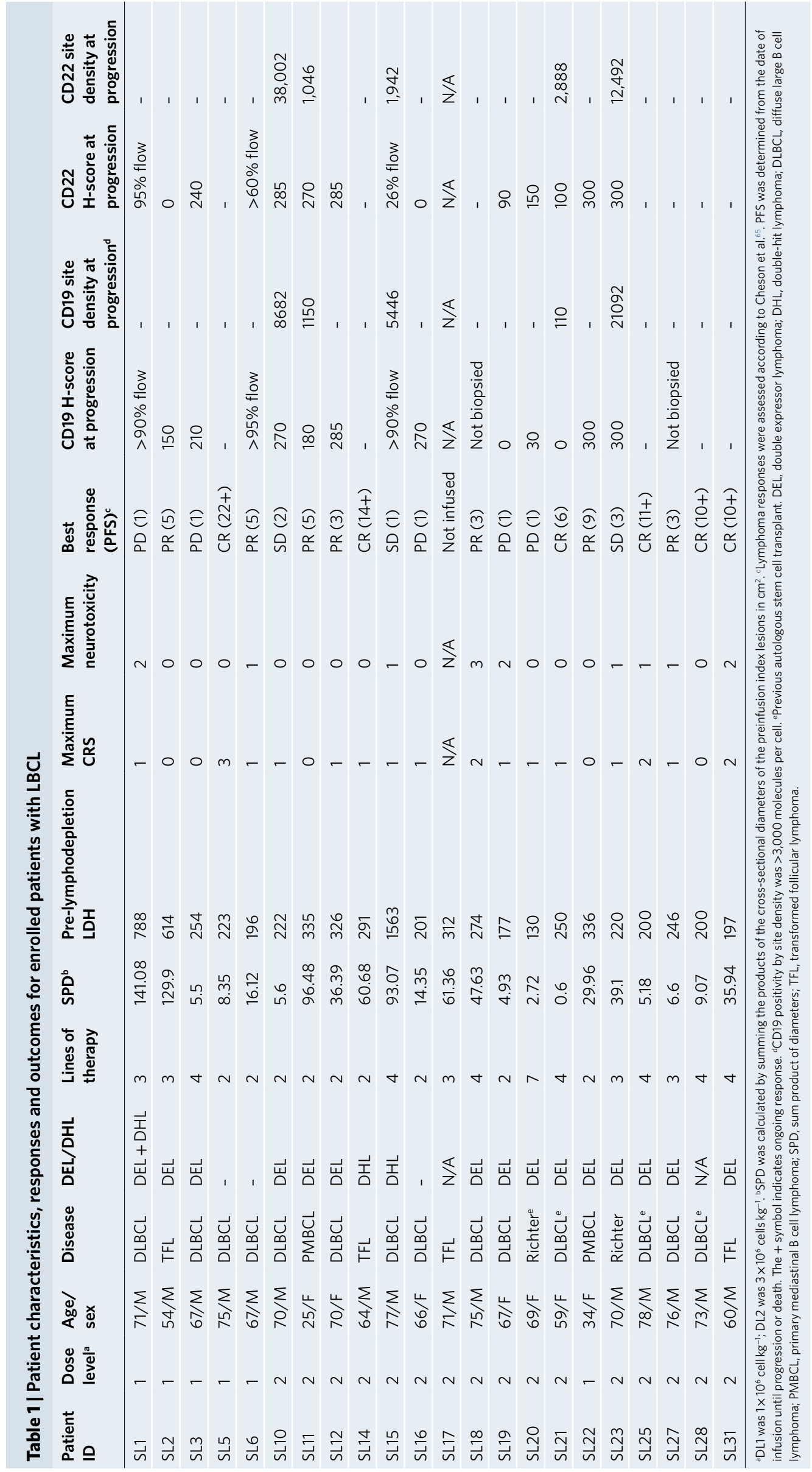



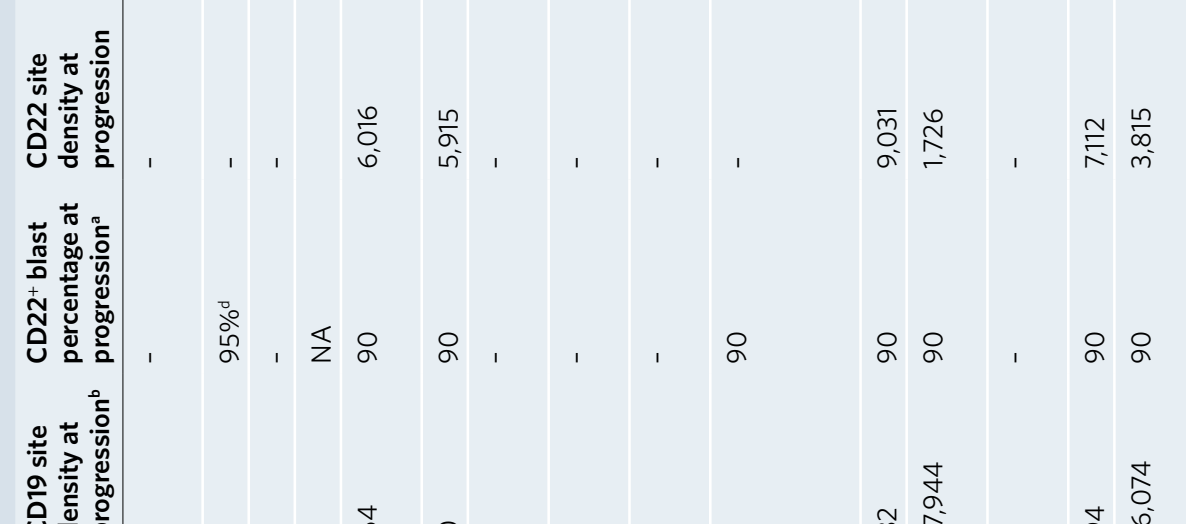

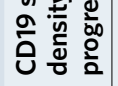

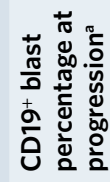

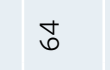

高, 总 :

$\therefore$

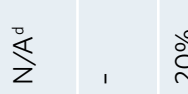

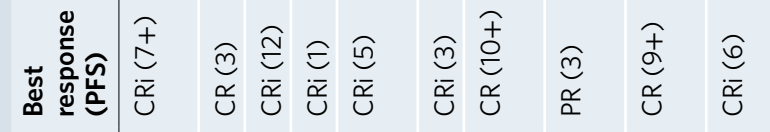

憘

웅

产

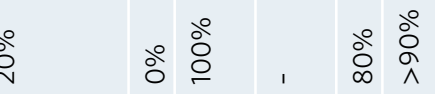

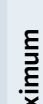

高

\section{年}


median overall survival was 11.8 months (95\% CI 5.5-NE) (Fig. 3i) and PFS was 5.8 months (95\% CI 2.6-NE) (Fig. 3j). Two patients proceeded to allogeneic stem cell transplant in CR and are in ongoing remission. Serial MRD analyses by next-generation sequencing (NGS) of immunoglobulin receptors from bone marrow revealed that $100 \%$ ( 5 out of 5 ) of patients with ongoing CR achieved persistent MRD-negative response, while $70 \%$ (7 out of 10) of progressors had persistence or rise in MRD before or at the time of morphological relapse (Extended Data Fig. 7).

In vivo quantification of CD19-22.BB.z CAR T cells. CD19-22. BB.z-CAR cells were detected in the blood by both flow cytometry and quantitative PCR (qPCR) and peaked between days 10-14 postinfusion (Fig. 3k,1). The median peak number of circulating CD19-22.BB.z cells measured by flow cytometry was $36 \mathrm{CAR}_{\mu} \mathrm{l}^{-1}$ (IQR = 13-136); by qPCR, it was $1,794(\mathrm{IQR}=509-4,315)$ copies of the CD19-22.BB.z transgene per 50 ng of genomic DNA. Peak expansion did not significantly differ between LBCL and B-ALL or dose level (Extended Data Fig. 8a,b). Higher expansion as measured by area under the curve (AUC) was associated with increased CRS and neurotoxicity (Extended Data Fig. 8c,d). Despite a predominance of $\mathrm{CD}^{+}$cells in the manufactured CD19-22.BB.z-CAR products, CD8 CD19-22.BB.z cells demonstrated greater expansion relative to CD4 as measured by both AUC and peak levels (Extended Data Fig. 8e,f) and as seen by a peak CD4:CD8 ratio $<1$ in most patients (Extended Data Fig. 8g). Analysis of exhaustion markers on $\mathrm{CAR}^{+}$cells within the product showed that $\mathrm{CD} 4^{+}$cells expressed higher levels of CD39 (ref. ${ }^{34}$ ) and programmed cell death protein 1 (PD-1) (Extended Data Fig. 8h,i), potentially providing a basis for diminished in vivo expansion of $\mathrm{CD} 4^{+}$compared to $\mathrm{CD} 8^{+} \mathrm{CAR}$ T cells.

Antigen expression in patients with progressive disease after CD19-22.BB.z-CAR. We next quantified CD19 and CD22 antigen expression at the time of progression after CD19-22.BB.z-CAR. Flow cytometry, as illustrated by patient SA24 (Fig. 4a), demonstrated that 5 of 10 patients with B-ALL with progression had negative or low CD19 expression with preserved CD22, using a $90 \%$ threshold (Fig. $4 \mathrm{~b}$ and Table 2). Paired pre- and posttherapy antigen quantification in four patients with B-ALL confirmed decreased CD19 expression in three patients with no change in CD22 expression density (Fig. 4c). In 14 patients with LBCL biopsied at progression, 3 were CD19-llo (Extended Data Fig. 9a) by IHC. CD22 expression was not required for trial enrollment and pretreatment CD22 expression was heterogenous (Extended Data Fig. 9b); three patients had CD22-1lo LBCL and two were undetermined. All 6 patients with a pretreatment CD22 H-score $>150$ maintained CD22 positivity. Eleven patients with B-ALL or LBCL had quantitative flow cytometry at progression; 6 patients had low CD19 with $\leq 3,000$ molecules per cell (Fig. 4 d), including 1 patient with LBCL with a CD19 H-score $>150$. The median CD22 expression in patients with low CD19 was approximately 6,000 molecules per cell. Overall, 4 out of 14 (29\%) patients with LBCL were CD19-/lo at progression after therapy with CD19-22.BB.z-CAR (Extended Data Fig. 9a and Fig. 4d).

CD19-22.BB.z-CAR $T$ secretes less cytokine when stimulated through the CD22 scFv. The pattern of $\mathrm{CD} 19^{-/ 10}$ relapse with CD22 preservation suggested that T cells expressing the CD19-22. BB.z-CAR exert significant immune pressure against CD19 but not against CD22. In vitro models demonstrated that CD19-22. BB.z-CAR was active against CD19/CD22 ${ }^{+}$cell lines (Extended Data Fig. 10a). To address this, we compared the relative potency of the signal delivered via the $\mathrm{CD} 19 \mathrm{scFv}$ versus the $\mathrm{CD} 22 \mathrm{scFv}$ within the CD19-22.BB.z-CAR (Fig. 4e) using single-cell assays. Using samples from 11 manufactured cell products, we measured the intracellular cytokine secretion (ICS) of CD19-22.BB.z-CAR T cells after coculture with double-positive NALM6 (N6-CD19/22, approximately 20,000 CD19 molecules per cell and approximately 50,000 CD22 molecules per cell), NALM6 where CD22 had undergone knockout via CRISPR-Cas9 (N6-CD19, approximately 20,000 CD19 molecules per cell, 0 CD22 molecules per cell), NALM6 where CD19 had undergone knockout via CRISPR-Cas9 (N6-CD22, approximately 40,000 CD22 molecules per cell, 0 CD19 molecules per cell) or NALM6 with both CD19 and CD22 genetically deleted (N6-double knockout) (Fig. 4f). These site densities were higher than those seen in patient samples ${ }^{21,28,35}$ and have been associated with high levels of cytokine secretion in in vitro models ${ }^{21}$.

Using ICS, CD19-22.BB.z-CAR manufactured products appeared activated as measured by CD69 expression after coculture with N6-CD19 and less activated with N6-CD22 $(P<0.0001)$. Higher expression with N6-CD19 compared with N6-CD22 was seen with CD107 $(P<0.0001)$, tumor necrosis factor- $\alpha(\mathrm{TNF} \alpha)$ $(P<0.0001)$, interleukin-2 (IL-2) $(P<0.0001)$ and interferon- $\gamma$ $($ IFN- $\gamma)(P<0.0001)$ (Fig. 4g).

Decreased PSI and ICS resulting from CD22 as opposed to CD19 stimulation suggested decreased potency of the CD22 scFV. We next compared the activity of the CD22 scFv of CD19-22. BB.z-CAR against the identical scFV in a monospecific CD22. BB.z-CAR (Fig. 4h) ${ }^{22,31,36}$. We tested good manufacturing practice (GMP)-manufactured CD22.BB.z-CAR T cell products $(n=5)$ from patients with relapsed/refractory LBCL or B-ALL enrolled on an ongoing clinical trial (NCT04088890) against N6-double knockout and N6-CD22. The mean fluorescence intensity of monospecific CAR on T cells was higher than that of the bispecific CAR (Extended Data Fig. 10b,c). We found that CD19-22. BB.z-CAR and CD22.BB.z-CAR T cells manifested similar levels of activation as measured by CD69 expression and IFN- $\gamma$ and CD107 secretion (Fig. 4i). However, CD22.BB.z-CAR T cells demonstrated significantly higher levels of TNF- $\alpha(P=0.0063)$ and IL-2 $(P=0.0002)$ secretion compared to CD19-22.BB.z-CAR T cells. Similarly, single-cell cytokine secretion using the IsoPlexis platform showed a higher PSI for CD22.BB.z-CAR T cells compared to CD19-22.BB.z-CAR in the presence of N6-CD22 $(n=4$; Fig. 4j). Single-cell cytokine secretion was assessed with the 32-plex

Fig. 3 | CD19-22.BB.z-CAR is active in both LBCL and B-ALL. a, Swimmer plot showing the duration of remission and ongoing responses in patients with lymphoma $(n=21)$. Five patients had an increasing depth of response from 1 to 3 months postinfusion $\mathbf{b}$, PET scans for patient S2 showing partial remission at 1 month postinfusion with subsequent progression 6 months after infusion. $\mathbf{c}$, Lymphoma disease monitoring using ctDNA. After infusion of CD19-22. BB.z for patient SL02, disease burden continued to decrease; this was coincident with prolonged persistence of CD19-22.BBZ. d, Overall survival for 21 infused patients with LBCL. e, PFS for the cohort with lymphoma. f, Swimmer plot for the cohort with B-ALL $(n=17)$. Two patients received a consolidative allogeneic stem cell transplant (white star) g, PET scans for patient SA8, with large bulk disease preinfusion that improved to a CR 6 months postinfusion. h, Disease monitoring of patient SA8 using cellular-based NGS with sensitivity of $10^{-6}$ demonstrates increasing disease control over time coinciding with improving PET response and ongoing persistence of CD19-22.BB.z. i, Overall survival of 17 infused patients with B-ALL. j, PFS for the cohort with B-ALL. k, Absolute number of circulating CD4 and CD8 CD19-22.BB.z CAR T cells after infusion as measured by flow cytometry ( $n=38$ autologous infused products). I, Number of circulating CD19-22.BB.z copies per $50 \mathrm{ng}$ of genomic DNA as measured by qPCR ( $n=33$ autologous infused products) showing initial expansion and persistence of CD19-22.BB.z as measured at 1 and 2 months (days 35-75 postinfusion), 3 months (days 76-120) and 6 months after infusion (days 120-200). 
human pane ${ }^{27}$ after stimulation of CD4 and CD8 CAR ${ }^{+}$cells with the N6-CD19/22, N6-double knockout, N6-CD19 or N6-CD22 N6 lines $(n=7)$. Overall, CD19 stimulation with N6-CD19 yielded a higher polyfunctional strength index (PSI) testing $\mathrm{CD} 8 \mathrm{CAR}^{+}$ products $(P=0.04)$ compared to N6-CD22 stimulation (Fig. 4j). Together, these data demonstrate that both CD19 and CD22 scFvs a

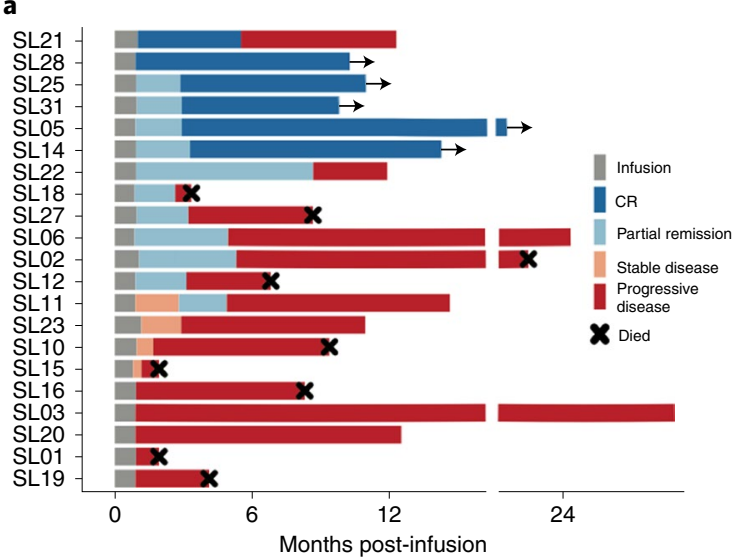

b
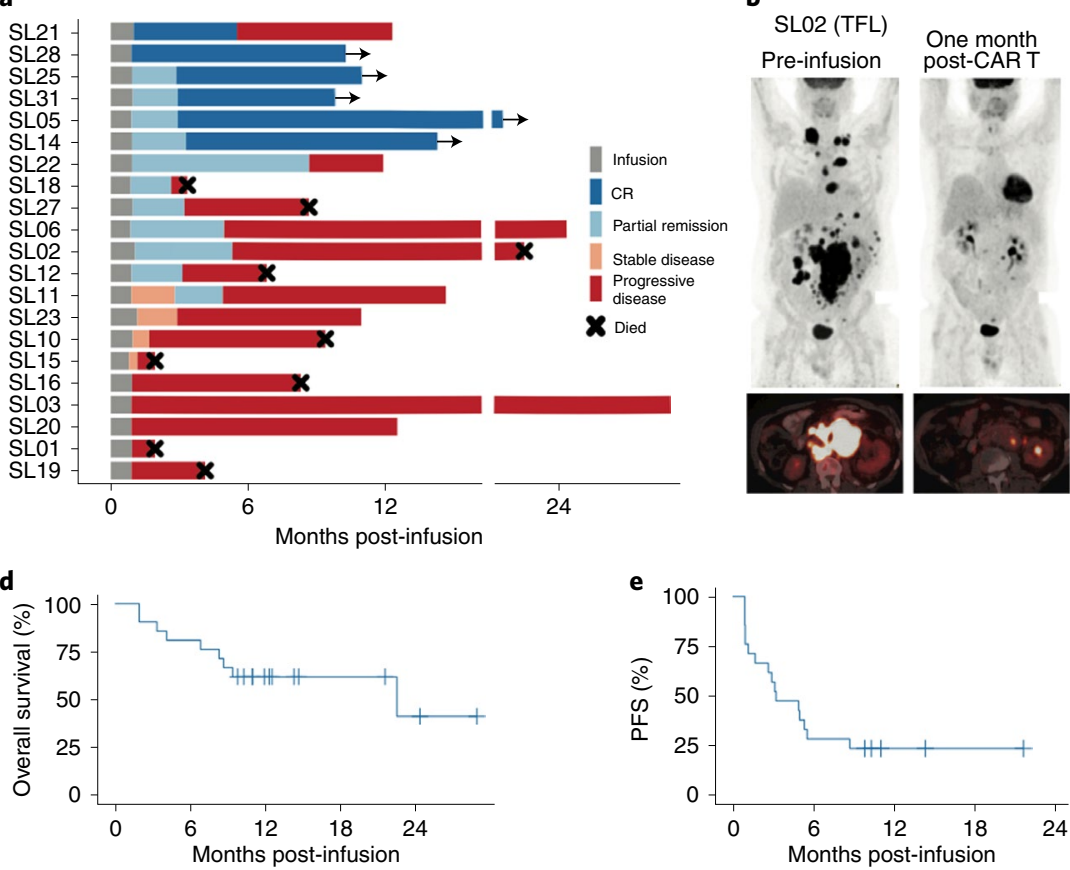

f

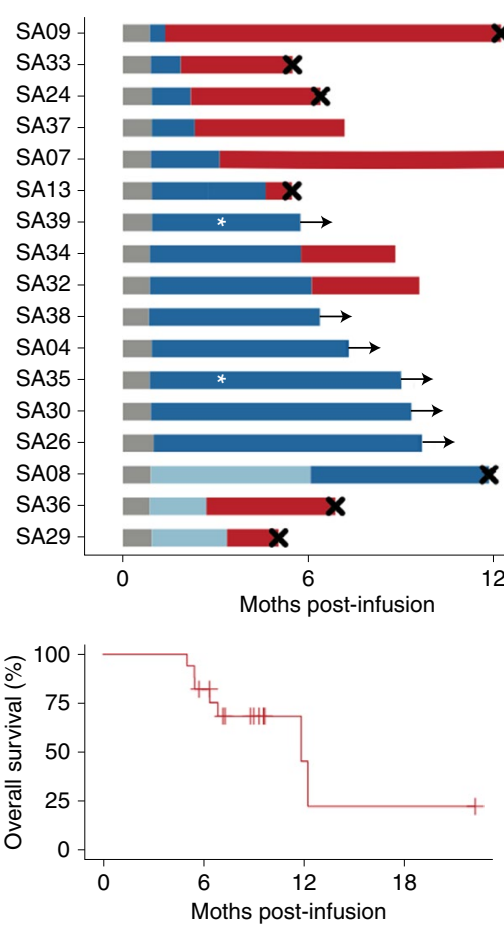

k

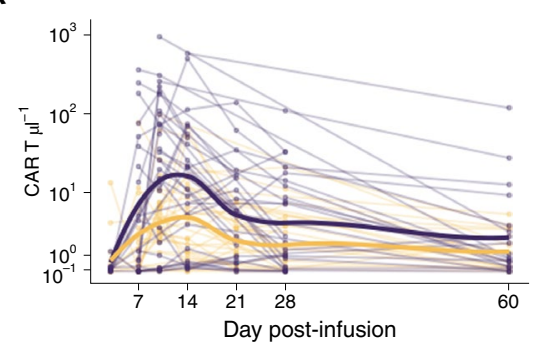

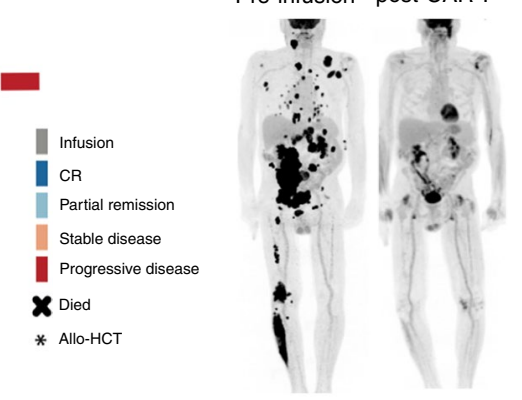

g SA08 (B-ALL) Pre-infusion 24
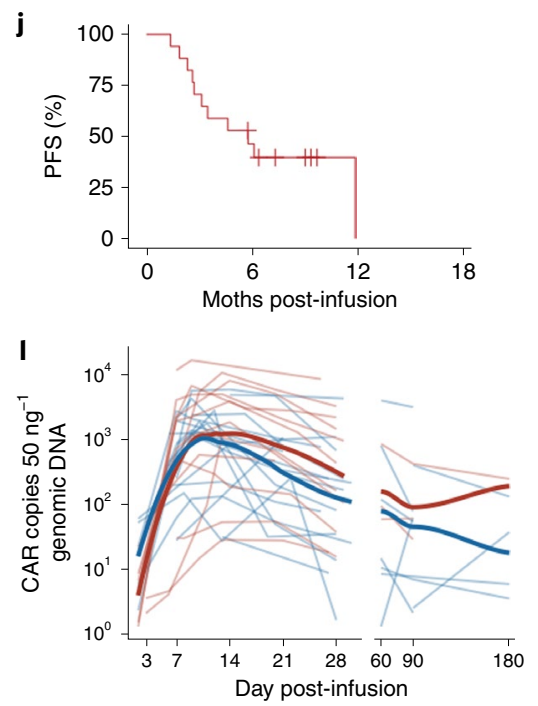

c

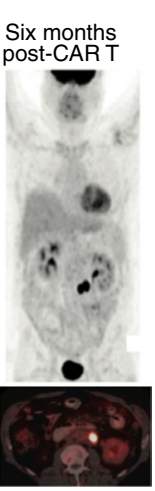

SL02 infusion no. 1

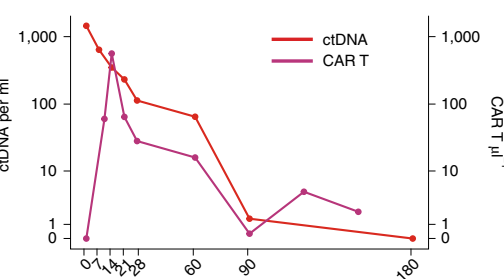

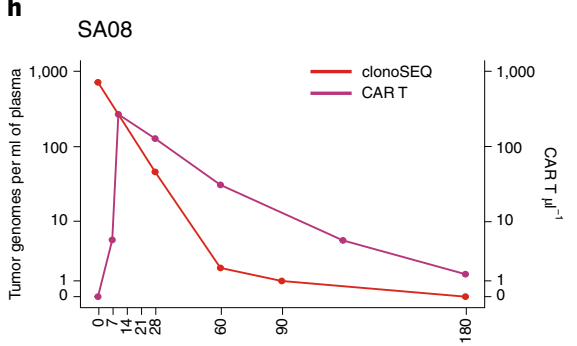


contained within CD19/22.BB.z-CAR can signal in response to target recognition but cytokine levels induced after CD22 scFv ligation in the bispecific CAR are lower compared to CD19 scFv ligation. In contrast, $\mathrm{CD} 22 \mathrm{scFv}$ ligation in the monospecific CAR resulted in higher levels of cytokines than the bispecific CD22 scFV. The reduced potency of the CD22 scFV provides a mechanism for
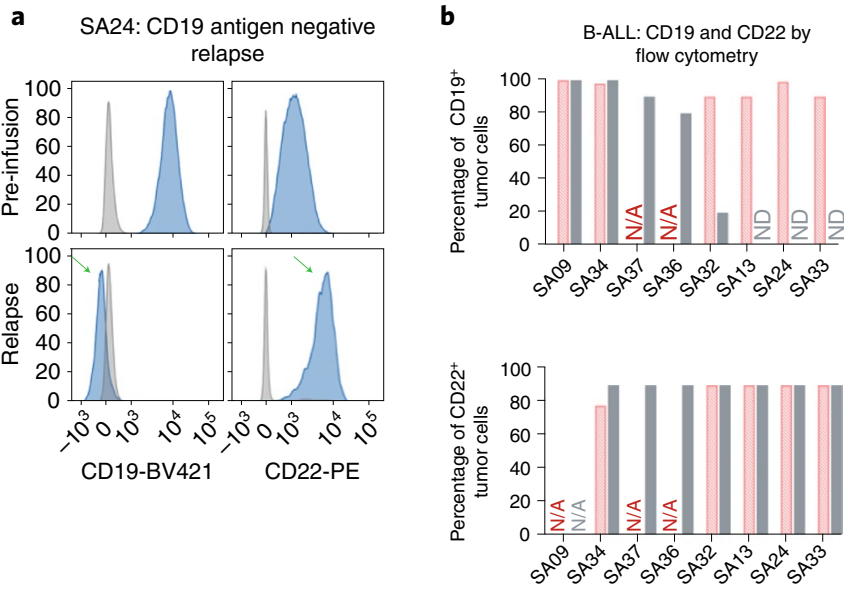

$\square$ Pre-CAR19/22
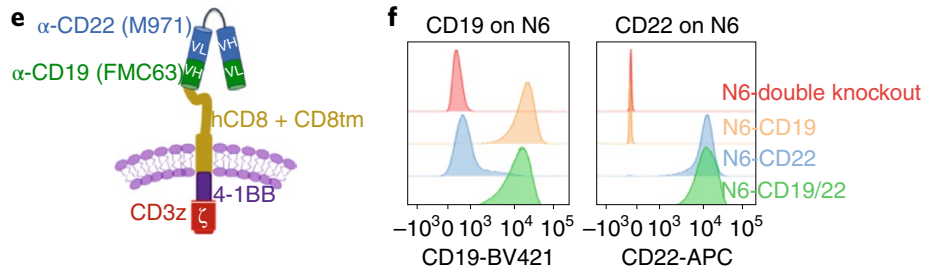

h

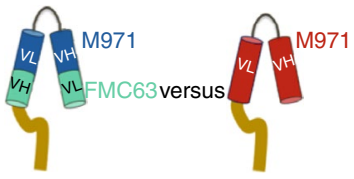

g

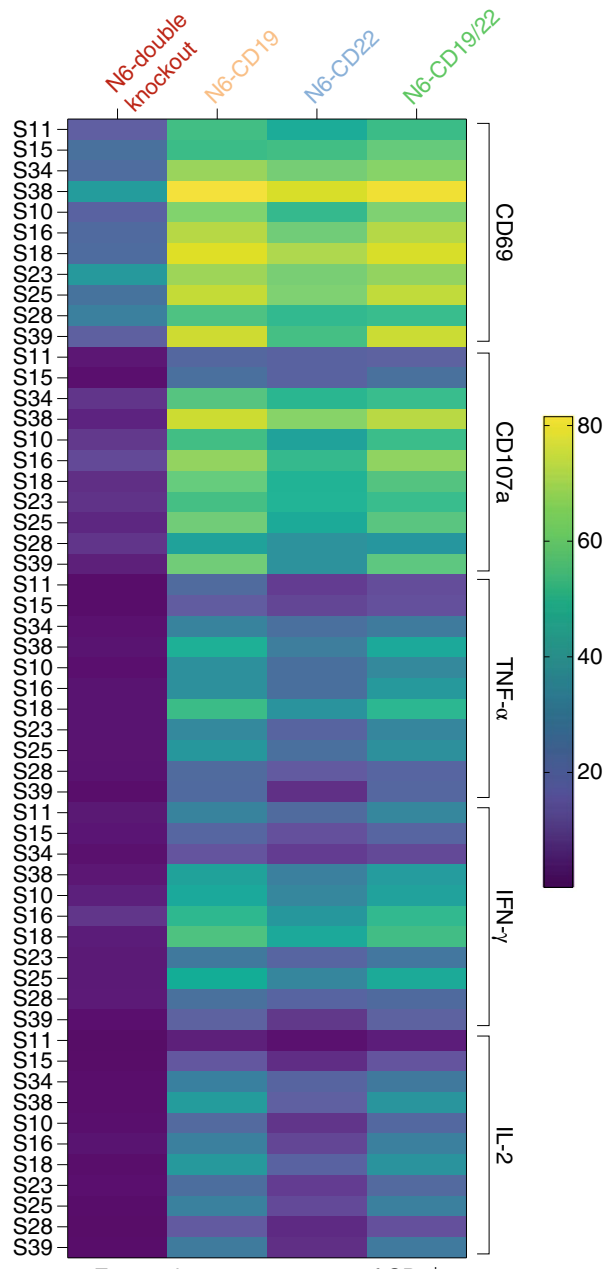

Expression as percentage of $\mathrm{CD}^{+}$ j

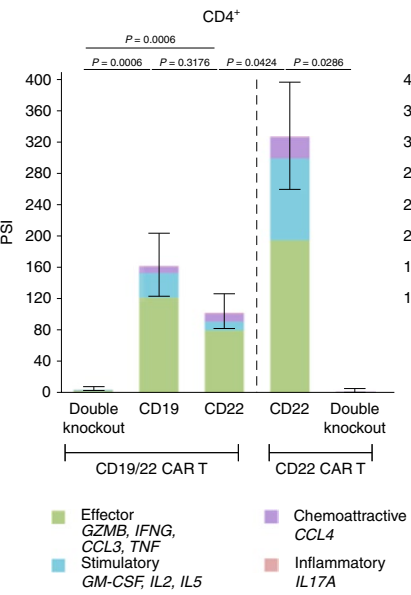

\begin{tabular}{|c|c|c|c|c|c|}
\hline \multicolumn{6}{|c|}{$\begin{array}{l}\text { Pre- and post-CD19/22 CAR } \\
\text { antigen density }\end{array}$} \\
\hline 15,099 & 17,945 & 3,589 & 1,726 & SA34 & $\begin{array}{l}10^{\circ} \\
-10^{4}\end{array}$ \\
\hline 20,479 & 82 & 6,232 & 9,031 & SA33 & $10^{3}$ \\
\hline 9,621 & 64 & 9,466 & 6,016 & SA13 & $10^{2}$ \\
\hline 8,018 & 0 & 1,264 & 5,915 & SA24 & $10^{1}$ \\
\hline Pre & elaps & Pre & Rel & & \\
\hline & & & & & \\
\hline
\end{tabular}

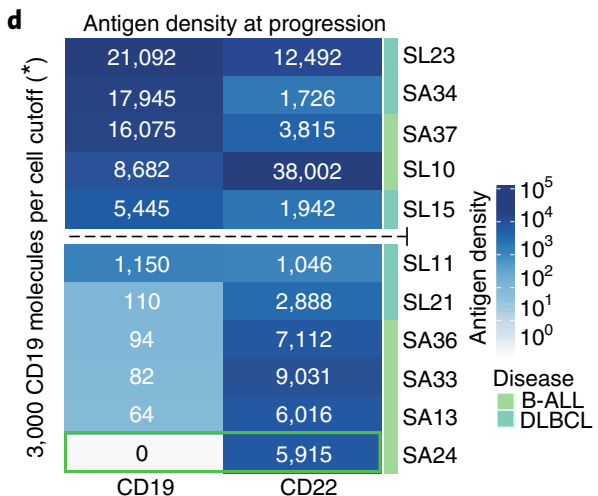

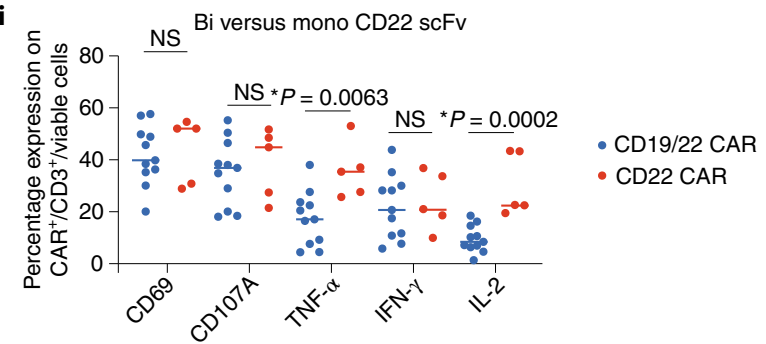
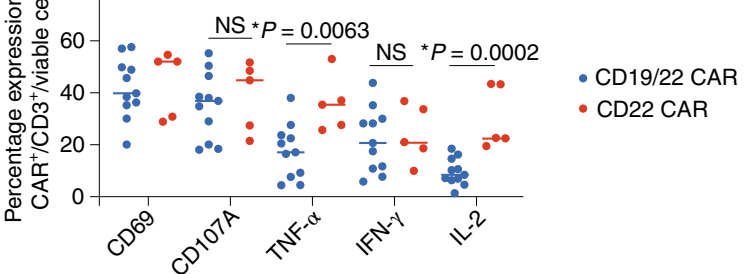

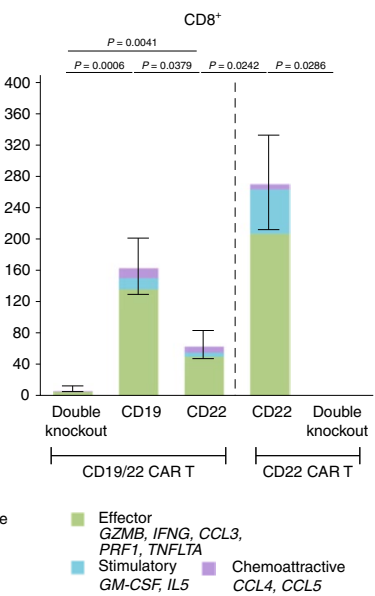


Fig. 4 | CD19 negative relapse with preserved CD22 site density after CD19-22.BB.z-CAR and diminished CAR T functionality against CD22. a, Antigen density of patient S24 demonstrating both CD19 and CD22 expression preCD19-22.BB.z (top) with loss of CD19 and preservation of CD22 at progression (bottom, green arrows). b, CD19 and CD22 assessment by conventional flow cytometry in patients with B-ALL pretreatment and postprogression demonstrates CD19 loss with CD22 preservation. c, In B-ALL, 3 of 4 patients with antigen quantification pre- and postCD19-22-CD.BB.z demonstrated loss of CD19 expression associated with preserved CD22 expression. d, CD19 and CD22 antigen density at progression ( $n=11$ patients) after CD19-22.BB.z, with patient S24 highlighted in green. Six patients had $<1,150$ CD19 molecules per cell with a median CD22 of approximately 6,000 molecules per cell. Dashed line denotes the cutoff at 3,000 CD19 molecules per cell. e, Schematic of CD19-22.BB.z bispecific CAR, displaying the loop structure. f, Histogram of CD19 and CD22 expression on NALM6 lines tested in $\mathbf{g}-\mathbf{j}$. g, ICS heatmap representing the secretion or expression of CD69, CD107a, TNF- $\alpha$, IFN- $\gamma$ and IL-2 from CD1922.BB.z infusion products ( $n=11$ individual products) stimulated with the NALM6 tumors lines from $\mathbf{f}$. The heatmap shows greater activation and secretion of cytokines with stimulation with N6-CD19 and N6-CD19/22 lines versus N6-CD22 stimulation. $\mathbf{h}$, Schematic of bispecific CD19-22.BB.z versus monospecific CD22.BB.z. i, ICS stimulation of CD19-22.BB.z $(n=11)$ versus monospecific CD22.BB.z $(n=5)$ CAR products against the CD22 ${ }^{\text {high }}$ cell line shows increased cytokine secretion of IL-2 and TNF- $\alpha$ through the monospecific CAR22.BB.z (two-tailed $t$-test). $\mathbf{j}$, Using the single-cell IsoPlexis platform, stimulation of clinical products ( $n=7$ individual products) with N6-CD19 showed a higher PSI compared to N6-CD22. The CD22 scFV on the bispecific CAR had lower PSI compared to the monospecific CAR22.BB.z ( $n=4$ individual products) when stimulated with N6-CD22 (Mann-Whitney U-test).

relapse with CD19-/lo disease with preserved CD22 expression in the population studied here.

\section{Discussion}

CD19 loss has been frequently identified after CAR19 in B-ALL across numerous studies incorporating variable scFvs and costimulatory domains $s^{5-7,15,16,37,38}$. In LBCL, case reports have also described CD19 loss after CAR19 ${ }^{35,39-41}$ but a systematic analysis of CD19 expression after CAR19 in LBCL has not been conducted. In this study, we evaluated CD19 expression in 44 patients with LBCL treated with axi-cel at our institution and found that pretreatment quantitative flow cytometry was more sensitive than conventional IHC in identifying lower antigen levels associated with future progression. Quantitative flow cytometry could serve as a predictive biomarker in characterizing antigen modulation as a mechanism of resistance to CAR therapeutics.

Several studies have demonstrated the efficacy of simultaneous targeting multiple antigens in preclinical models using a variety of CAR configurations to endow multi-specific antigen recognition $^{22,42-46}$. Early clinical trial results involving tandem CAR T cells targeting CD19 and CD20 have shown promising results with low reported rates of CD19 loss at the time of progression ${ }^{47,48}$. The CD19-22.BB.z-CAR used in this trial is a single molecule consisting of two scFvs engineered in a loop orientation ${ }^{22}$, which demonstrated activity in vitro and in xenograft models ${ }^{32}$. The clinical data presented in this study demonstrated that CD19-22.BB.z-CAR T cells were clinically active in both B-ALL, with $82 \%$ achieving an $\mathrm{MRD}^{-}$ CR, and in LBCL with an ORR of $62 \%$. Toxicity was low: 5 and $11 \%$ experienced grade $\geq 3$ CRS or neurotoxicity, respectively. Although we observed relapses with absent or low CD19 expression, a definitive determination of whether these rates are reduced compared to those observed with monospecific CAR19 therapeutics cannot be determined in this single-arm trial given the limited data available regarding antigen ${ }^{-/ l o}$ relapse and the wide variability in antigen loss reported across studies.

However, the antigen expression pattern observed at relapse in B-ALL was consistent with CD19-22.BB.z-CAR delivering significant immune pressure on the CD19 antigen, whereas the lack of decrease or loss of CD22 expression suggests more limited immune pressure on the CD22 target. Experience with monospecific CD22-CAR T cells clearly demonstrated resistance associated with $\mathrm{CD} 22^{\text {lo }} \mathrm{B}-\mathrm{ALL}$, which was not observed in this trial ${ }^{22}$. In LBCL, $\mathrm{CD} 22^{\text {lo }}$ disease at relapse was seen; however, CD22 expression before therapy was heterogeneous and absent in some. Our data show that CD22 scFv ligation in the bispecific CAR provided less cytokine secretion compared to CD19 scFv. These results suggest cytokine production could be a clinically meaningful product quality attribute to predict CAR potency in vivo and illustrate the challenges of delivering equivalent potency across targets in the context of multi-specific chimeric antigen receptors.
At present, much of CAR engineering is empiric and multiple approaches to simultaneous targeting of CD19 and CD22 are currently under study ${ }^{32,49-52}$. Engineering an optimal monospecific CAR is dependent on numerous factors, including prevention of tonic signaling, ${ }^{53}$ optimizing hinge length, ${ }^{54,55}$ hinge/transmembrane domain $^{21}$ and optimizing the distance between the target epitope and tumor cell membrane ${ }^{27,56,57}$. In human trials, numerous chimeric antigen receptors targeting CD22 have demonstrated that small alterations in structure can result in loss of activity ${ }^{49,58}$. In the context of a tandem CAR (linked scFvs), optimal engineering also requires engineering an optimal bispecific receptor ${ }^{59,60}$, which may be particularly challenging for the CD22 target. When compared to a monospecific CAR with the same scFV, the CD19-22.BB.z-CAR demonstrated decreased TNF- $\alpha$ and IL- 2 secretion. The threshold for CAR T cell IL-2 secretion has previously been shown to be higher than that of IFN- $\gamma^{20}$ and may better discriminate CAR efficacy. Our results suggest that engineering iterations should be guided by careful studies of single-cell CAR polyfunctionality incorporating cytokine production as a critical quality attribute.

We also observed a significant incidence of $\mathrm{CD} 19^{+}$relapse, as reported in many previous trials of CAR19 for B-ALL and $\mathrm{LBCL}^{61}$, suggesting that potency toward the CD19 target may also be insufficient in some patients. Improvements in CAR manufacturing could prevent antigen ${ }^{+}$resistance, which likely results from $\mathrm{T}$ cell failure. Defined composition of CD4:CD8 CAR cells and preferential administration of $\mathrm{T}$ cell subsets, such as $\mathrm{T}_{\mathrm{CM}}{ }^{62,63}$, have been hypothesized to mediate optimal CAR activity ${ }^{1}$. We found our manufacturing process selectively enriched for $\mathrm{T}_{\mathrm{CM}}$ and $\mathrm{T}_{\mathrm{SCM}}$ with low $\mathrm{T}_{\mathrm{EMRA}}$ but biased the final product toward $\mathrm{CD} 4$ predominance. Furthermore, CD4 cells produced with our process expressed higher levels of CD39 and PD-1, which are associated with exhaustion, suggesting that the manufacturing process may not generate an optimal final product composition. Due to these findings, we paused trial enrollment to modify our manufacturing process to attain a more balanced CD4:CD8 ratio, which may also allow CD4 CAR cells to maintain a less exhausted phenotype.

In summary, this work provides evidence that antigen ${ }^{-/ l o}$ escape is a major pathway of resistance after CAR19 therapy for LBCL and quantitative antigen density in LBCL correlates with outcomes after CAR T cell therapy. Using a bispecific CAR capable of simultaneously recognizing CD22 and CD19, we demonstrated safety and impressive clinical activity in B-ALL. The 6-month PFS in LBCL $(29 \%, 95 \%$ CI $12-48 \%)$ in this trial was similar to tisagenlecleucel ${ }^{64}$. Therefore, the lymphoma arm was closed in this study with enrollment ongoing in patients with B-ALL. Resistance to the bispecific $\mathrm{CAR}$ was associated with $\mathrm{CD} 19^{+} \mathrm{CD} 22^{+}$relapse, probably reflecting intrinsic limitations of CAR T cells, as well as CD19-/lo but CD22 $2^{+}$ relapse, implying inadequate immune pressure on the CD22 target. Our data also illustrate the value of cytokine production as a key product quality attribute for credentialing the potency of CAR 
T cells. Future work is needed to optimize multi-specific targeting by CAR T cells to improve the efficacy of this class of therapeutics both in $\mathrm{B}$ cell malignancies and other solid and liquid cancers.

\section{Online content}

Any methods, additional references, Nature Research reporting summaries, source data, extended data, supplementary information, acknowledgements, peer review information; details of author contributions and competing interests; and statements of data and code availability are available at https://doi.org/10.1038/ s41591-021-01436-0.

Received: 29 September 2020; Accepted: 9 June 2021;

Published online: 26 July 2021

\section{References}

1. Turtle, C. J. et al. CD19 CAR-T cells of defined CD4+CD8 ${ }^{+}$composition in adult B cell ALL patients. J. Clin. Invest. 126, 2123-2138 (2016).

2. Locke, F. L. et al. Long-term safety and activity of axicabtagene ciloleucel in refractory large B-cell lymphoma (ZUMA-1): a single-arm, multicentre, phase 1-2 trial. Lancet Oncol. 20, 31-42 (2019).

3. Neelapu, S. S. et al. Axicabtagene ciloleucel CAR T-cell therapy in refractory large B-cell lymphoma. N. Engl. J. Med. 377, 2531-2544 (2017).

4. Schuster, S. J. et al. Tisagenlecleucel in adult relapsed or refractory diffuse large B-cell lymphoma. N. Engl. J. Med. 380, 45-56 (2019).

5. Hay, K. A. et al. Factors associated with durable EFS in adult B-cell ALL patients achieving MRD-negative CR after CD19 CAR T-cell therapy. Blood 133, 1652-1663 (2019).

6. Maude, S. L. et al. Tisagenlecleucel in children and young adults with B-cell lymphoblastic leukemia. N. Engl. J. Med. 378, 439-448 (2018).

7. Park, J. H. et al. Long-term follow-up of CD19 CAR therapy in acute lymphoblastic leukemia. N. Engl. J. Med. 378, 449-459 (2018).

8. Nastoupil, L. J. Standard-of-care axicabtagene ciloleucel for relapsed or refractory large B-cell lymphoma: results from the US Lymphoma CAR T Consortium. J. Clin. Oncol. 38, 3119-3128 (2020).

9. Abramson, J. S. et al. Pivotal safety and efficacy results from Transcend NHL 001, a multicenter phase 1 study of lisocabtagene maraleucel (liso-cel) in relapsed/refractory (R/R) large B cell lymphomas. Blood 134, 241 (2019).

10. Lee, D. W. et al. T cells expressing CD19 chimeric antigen receptors for acute lymphoblastic leukaemia in children and young adults: a phase 1 dose-escalation trial. Lancet 385, 517-528 (2015).

11. Liu, E. et al. Use of CAR-transduced natural killer cells in CD19-positive lymphoid tumors. N. Engl. J. Med. 382, 545-553 (2020).

12. Majzner, R. G. \& Mackall, C. L. Tumor antigen escape from CAR T-cell therapy. Cancer Discov. 8, 1219-1226 (2018).

13. Bagashev, A. et al. CD19 alterations emerging after CD19-directed immunotherapy cause retention of the misfolded protein in the endoplasmic reticulum. Mol. Cell. Biol. 38, e00383-18 (2018).

14. Asnani, M. et al. Retention of CD19 intron 2 contributes to CART-19 resistance in leukemias with subclonal frameshift mutations in CD19. Leukemia 34, 1202-1207 (2020).

15. Orlando, E. J. et al. Genetic mechanisms of target antigen loss in CAR19 therapy of acute lymphoblastic leukemia. Nat. Med. 24, 1504-1506 (2018).

16. Sotillo, E. et al. Convergence of acquired mutations and alternative splicing of CD19 enables Resistance to CART-19 Immunotherapy. Cancer Discov. 5, 1282-1295 (2015).

17. Majzner, R. G. et al. CAR T cells targeting B7-H3, a pan-cancer antigen, demonstrate potent preclinical activity against pediatric solid tumors and brain tumors. Clin. Cancer Res. 25, 2560-2574 (2019).

18. Watanabe, $K$. et al. Target antigen density governs the efficacy of anti-CD20-CD28-CD3 $\zeta$ chimeric antigen receptor-modified effector $\mathrm{CD} 8^{+}$ T cells. J. Immunol. 194, 911-920 (2015).

19. Hombach, A. A. et al. Superior therapeutic index in lymphoma therapy: $\mathrm{CD} 30^{+} \mathrm{CD} 34^{+}$hematopoietic stem cells resist a chimeric antigen receptor T-cell attack. Mol. Ther. 24, 1423-1434 (2016).

20. Walker, A. J. et al. Tumor antigen and receptor densities regulate efficacy of a chimeric antigen receptor targeting anaplastic lymphoma kinase. Mol. Ther. 25, 2189-2201 (2017).

21. Majzner, R. G. et al. Tuning the antigen density requirement for CAR T-cell activity. Cancer Discov. 10, 702-723 (2020).

22. Fry, T. J. et al. CD22-targeted CAR T cells induce remission in B-ALL that is naive or resistant to CD19-targeted CAR immunotherapy. Nat. Med. 24, $20-28$ (2018).

23. Cohen, A. D. et al. B cell maturation antigen-specific CAR T cells are clinically active in multiple myeloma. J. Clin. Invest. 129, 2210-2221 (2019).
24. Oak, J. et al. Target antigen downregulation and other mechanisms of failure after axicabtagene ciloleucel (CAR19) therapy. Blood 132, 4656 (2018).

25. Neelapu, S. S. et al. CD19-loss with preservation of other B cell lineage features in patients with large B cell lymphoma who relapsed post-axi-cel. Blood 134, 203 (2019).

26. Shah, N. N. et al. Characterization of CD22 expression in acute lymphoblastic leukemia. Pediatr. Blood Cancer 62, 964-969 (2015).

27. Haso, W. et al. Anti-CD22-chimeric antigen receptors targeting B-cell precursor acute lymphoblastic leukemia. Blood 121, 1165-1174 (2013).

28. Tedder, T. F., Poe, J. C. \& Haas, K. M. CD22: a multifunctional receptor that regulates B lymphocyte survival and signal transduction. Adv. Immunol. 88, $1-50$ (2005)

29. Raponi, S. et al. Flow cytometric study of potential target antigens (CD19, CD20, CD22, CD33) for antibody-based immunotherapy in acute lymphoblastic leukemia: analysis of 552 cases. Leuk. Lymphoma $\mathbf{5 2}$ 1098-1107 (2011).

30. Olejniczak, S. H., Stewart, C. C., Donohue, K. \& Czuczman, M. S. A quantitative exploration of surface antigen expression in common B-cell malignancies using flow cytometry. Immunol. Invest. 35, 93-114 (2006).

31. Shah, N. N. et al. CD4/CD8 T-cell selection affects chimeric antigen receptor (CAR) T-cell potency and toxicity: updated results from a phase I anti-CD22 CAR T-cell trial. J. Clin. Oncol. 38, 1938-1950 (2020).

32. Qin, H. et al. Preclinical development of bivalent chimeric antigen receptors targeting both CD19 and CD22. Mol. Ther. Oncolytics 11, 127-137 (2018).

33. Frank, M. J. et al. Optimizing circulating tumor DNA based assessments in patients with large B-cell lymphoma undergoing axicabtagene ciloleucel. J. Clin. Oncol. https://doi.org/10.1200/JCO.21.00377 (2021).

34. Weber, E. W. et al. Transient rest restores functionality in exhausted CAR-T cells through epigenetic remodeling. Science 372, eaba1786 (2021).

35. Yu, H. et al. Repeated loss of target surface antigen after immunotherapy in primary mediastinal large B cell lymphoma. Am. J. Hematol. 92, E11-E13 (2017).

36. Baird, J. H. et al. CD22-directed CAR T-cell therapy induces complete remissions in CD19-directed CAR-refractory large B-cell lymphoma. Blood 137, 2321-2325 (2021).

37. Gardner, R. et al. Acquisition of a CD19-negative myeloid phenotype allows immune escape of MLL-rearranged B-ALL from CD19 CAR-T-cell therapy. Blood 127, 2406-2410 (2016).

38. Jacoby, E. et al. CD19 CAR immune pressure induces B-precursor acute lymphoblastic leukaemia lineage switch exposing inherent leukaemic plasticity. Nat. Commun. 7, 12320 (2016)

39. Schuster, S. J. et al. Chimeric antigen receptor $\mathrm{T}$ cells in refractory B-cell lymphomas. N. Engl. J. Med. 377, 2545-2554 (2017).

40. Shalabi, H. et al. Sequential loss of tumor surface antigens following chimeric antigen receptor T-cell therapies in diffuse large B-cell lymphoma. Haematologica 103, e215-e218 (2018).

41. Ali, S. A. et al. T cells expressing an anti-B-cell maturation antigen chimeric antigen receptor cause remissions of multiple myeloma. Blood $\mathbf{1 2 8}$, 1688-1700 (2016).

42. Hegde, M. et al. Tandem CAR T cells targeting HER2 and IL13R $\alpha 2$ mitigate tumor antigen escape. J. Clin. Invest. 126, 3036-3052 (2016).

43. Bielamowicz, K. et al. Trivalent CAR T cells overcome interpatient antigenic variability in glioblastoma. Neuro Oncol. 20, 506-518 (2018).

44. Zah, E. et al. Systematically optimized BCMA/CS1 bispecific CAR-T cells robustly control heterogeneous multiple myeloma. Nat. Commun. 11, 2283 (2020).

45. He, X. et al. Bispecific and split CAR T cells targeting CD13 and TIM3 eradicate acute myeloid leukemia. Blood 135, 713-723 (2020).

46. Schmidts, A. et al. Rational design of a trimeric APRIL-based CAR-binding domain enables efficient targeting of multiple myeloma. Blood Adv. $\mathbf{3}$ 3248-3260 (2019).

47. Shah, N. N. et al. Bispecific anti-CD20, anti-CD19 CAR T cells for relapsed B cell malignancies: a phase 1 dose escalation and expansion trial. Nat. Med. 26, 1569-1575 (2020)

48. Tong, C. et al. Optimized tandem CD19/CD20 CAR-engineered T cells in refractory/relapsed B-cell lymphoma. Blood 136, 1632-1644 (2020).

49. Gardner, R. A. et al. Efficacy of SCRI-CAR19x22 T cell product in B-ALL and persistence of anti-CD22 activity. J. Clin. Oncol. 38, 3035 (2020).

50. Fousek, K. et al. CAR T-cells that target acute B-lineage leukemia irrespective of CD19 expression. Leukemia 35, 75-89 (2021).

51. Dai, H. et al. Bispecific CAR-T cells targeting both CD19 and CD22 for therapy of adults with relapsed or refractory B cell acute lymphoblastic leukemia. J. Hematol. Oncol. 13, 30 (2020).

52. Amrolia, P. J. et al. Phase I study of AUTO3, a bicistronic chimeric antigen receptor (CAR) T-cell therapy targeting CD19 and CD22, in pediatric patients with relapsed/refractory B-cell acute lymphoblastic leukemia $(\mathrm{r} / \mathrm{r}$ B-ALL): Amelia study. Blood 134, 2620 (2019).

53. Lynn, R. C. et al. c-Jun overexpression in CAR T cells induces exhaustion resistance. Nature 576, 293-300 (2019). 
54. Guest, R. D. et al. The role of extracellular spacer regions in the optimal design of chimeric immune receptors: evaluation of four different scFvs and antigens. J. Immunother. 28, 203-211 (2005).

55. Hudecek, M. et al. Receptor affinity and extracellular domain modifications affect tumor recognition by ROR1-specific chimeric antigen receptor T cells Clin. Cancer Res. 19, 3153-3164 (2013).

56. Long, A. H., Haso, W. M. \& Orentas, R. J. Lessons learned from a highly-active CD22-specific chimeric antigen receptor. Oncoimmunology 2, e23621 (2013).

57. Li, D. et al. Persistent polyfunctional chimeric antigen receptor T cells that target glypican 3 eliminate orthotopic hepatocellular carcinomas in mice. Gastroenterology 158, 2250-2265.e20 (2020).

58. Singh, N. et al. Single chain variable fragment linker length regulates CAR biology and T cell efficacy. Blood 134, 247 (2019).

59. Grada, Z. et al. TanCAR: a novel bispecific chimeric antigen receptor for cancer immunotherapy. Mol. Ther. Nucleic Acids 2, e105 (2013).

60. Zah, E., Lin, M.-Y., Silva-Benedict, A., Jensen, M. C. \& Chen, Y. Y. T cells expressing CD19/CD20 bispecific chimeric antigen receptors prevent antigen escape by malignant B cells. Cancer Immunol. Res. 4, 498-508 (2016)

61. Spiegel, J. Y. et al. Outcomes of patients with large B-cell lymphoma progressing after axicabtagene ciloleucel therapy. Blood 137, 1832-1835 (2021).

62. Blaeschke, F. et al. Induction of a central memory and stem cell memory phenotype in functionally active $\mathrm{CD}^{+}$and $\mathrm{CD} 8^{+} \mathrm{CAR} \mathrm{T}$ cells produced in an automated good manufacturing practice system for the treatment of $\mathrm{CD}_{19^{+}}$acute lymphoblastic leukemia. Cancer Immunol. Immunother. 67, 1053-1066 (2018)
63. $\mathrm{Xu}, \mathrm{Y}$. et al. Closely related T-memory stem cells correlate with in vivo expansion of CAR.CD19-T cells and are preserved by IL-7 and IL-15. Blood 123, 3750-3759 (2014).

64. Pasquini, M. C. et al. Real-world evidence of tisagenlecleucel for pediatric acute lymphoblastic leukemia and non-Hodgkin lymphoma. Blood Adv. 4, 5414-5424 (2020).

65. Cheson, B. D. et al. Recommendations for initial evaluation, staging, and response assessment of Hodgkin and non-Hodgkin lymphoma: the Lugano classification. J. Clin. Oncol. 32, 3059-3067 (2014).

Publisher's note Springer Nature remains neutral with regard to jurisdictional claims in published maps and institutional affiliations.

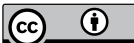

Open Access This article is licensed under a Creative Commons

Attribution 4.0 International License, which permits use, sharing, adaptation, distribution and reproduction in any medium or format, as long as you give appropriate credit to the original author(s) and the source, provide a link to the Creative Commons license, and indicate if changes were made. The images or other third party material in this article are included in the article's Creative Commons license, unless indicated otherwise in a credit line to the material. If material is not included in the article's Creative Commons license and your intended use is not permitted by statutory regulation or exceeds the permitted use, you will need to obtain permission directly from the copyright holder. To view a copy of this license, visit http://creativecommons. org/licenses/by/4.0/.

(C) The Author(s) 202 


\section{Methods}

Trial design. We conducted a single-center phase I clinical trial of CD19-22. BB.z-CAR in adult patients with relapsed/refractory LBCL and B-ALL at Stanford University Medical Center. The study was approved by the internal review board and registered with ClinicalTrials.gov (NCT03233854). A trial-specific safety monitoring committee was chartered for safety and trial conduct; it consisted of internal Stanford and external CAR T expert members. In addition, data monitoring was overseen by Stanford's institutional Data Safety Monitoring Committee. Informed written consent was provided by all patients in accordance with the Declaration of Helsinki (2013). The full clinical trial protocol is included in the Supplementary Information. The dose escalation phase enrolled both histologies in a single arm and permitted enrollment on three dose levels using a $3+3$ design: DL1 of $1 \times 10^{6}$ cells kg $^{-1}$; DL2 of $3 \times 10^{6} \mathrm{cells} \mathrm{kg}^{-1}$; and DL3 of $1 \times 10^{7}$ cells $\mathrm{kg}^{-1}$. Primary outcomes were feasibility of manufacture of CD19-22.BB.z-CAR as well as safety of CD19-22.BB.z-CAR. Feasibility of manufacture was defined as greater than $80 \%$ of products meeting the protocol-specified dose. Safety was defined by incidence and severity of DLTs at each of the dose levels tested; the maximal tolerated dose was defined as the dose level immediately below the dose level where incidence of DLTs was $>30 \%$. After dose escalation, disease-specific dose expansion cohorts could be enrolled at either the maximum tolerated dose or highest dose level tested. Secondary outcomes included efficacy of CD19-22. BB.z-CAR to induce clinical response at three months for LBCL and one month for B-ALL. A futility analysis was conducted after 15 individuals were enrolled at the recommended phase 2 dose. In LBCL, futility was defined as a $\leq 33 \%$ ORR at the 3 -month time point. In B-ALL, futility was defined as a $\leq 40 \%$ ORR at the 1-month time point. Exploratory objectives included the rate of $\mathrm{CD}^{-} 9^{-}$and/or $\mathrm{CD} 22^{-}$ relapse after CD19-22.BB.z-CAR, site density of CD19 and CD22 of malignant cells and expansion and persistence of CD19-22.BB.z-CAR after infusion.

Patient enrollment and eligibility. Patients eligible for leukapheresis were $\geq 18$ with LBCL or B-ALL relapsed or refractory after $\geq 2$ lines of therapy. If applicable, previous autologous or allogeneic HCT were considered as a line of therapy. Patients with LBCL must have received an anthracycline and an anti-CD20 monoclonal antibody as part of previous therapy. Transformed indolent lymphomas, including Richter transformation, were eligible. Measurable disease by PET/CT was required. For B-ALL, either morphological disease (including extramedullary disease) or detectable MRD with $<5 \%$ marrow blasts were acceptable; CNS involvement was allowed. Expression of CD19 on malignant cells was required by either flow cytometry or IHC. Previous anti-CD19-directed therapy, including previous CAR T, was allowed provided $<5 \%$ of circulating $\mathrm{T}$ cells expressed the previous CAR. At least two weeks or five half-lives, whichever was shorter, must have elapsed since any previous systemic therapy at the time of leukapheresis and all previous toxicities must be stable or recovered to grade 1 or lower. Adequate marrow function was required unless cytopenias were felt to be due to underlying leukemia/lymphoma: an absolute neutrophil count $\geq 750 \mu \mathrm{l}$, platelet count $\geq 50,000 \mu \mathrm{l}$ and absolute lymphocyte count $\geq 150 \mu \mathrm{l}$. Adequate organ function was defined as creatinine $\geq 2 \mathrm{mg} \mathrm{ml}^{-1}$, serum aspartate aminotransferase or alanine aminotransferase less than ten times the upper limit of normal, total bilirubin $\leq 1.5 \mathrm{mg} \mathrm{dl}^{-1}$, cardiac ejection fraction $\geq 45 \%$, no clinically significant electrocardiogram findings, no clinically significant pleural effusion and baseline oxygen saturation $\geq 92 \%$ on room air. Exclusion criteria included: history of previous malignancy within 3 years; presence of uncontrolled bacterial, viral or fungal infection or infection requiring intravenous antibiotics; known infection with human immunodeficiency virus, hepatitis $\mathrm{B}\left(\mathrm{HBsAg}^{+}\right)$ or hepatitis $\mathrm{C}$ (anti-hepatitis $\mathrm{C}$ virus ${ }^{+}$); CNS disorder impairing one's ability to evaluate neurotoxicity; history of autoimmune disease requiring systemic immunosuppression within previous 2 years; history of clinically significant cardiac disease within 12 months of enrollment.

After leukapheresis, bridging therapy was permitted at the investigator's discretion. Conditioning chemotherapy, consisting of fludarabine $30 \mathrm{mg} \mathrm{m}^{-2}$ and cyclophosphamide $500 \mathrm{mg} \mathrm{m}^{-2}$, was administered on day -5 through day -3 before infusion. Patients were enrolled to receive CD19-22.BB.z-CAR between 12 September 2017 and 19 November 2019. Data were locked as of 15 June 2020. Dose escalation and production feasibility were determined by consecutive patients irrespective of disease type. After dose escalation, separate LBCL and B-ALL cohorts were expanded to treat 15 patients in each cohort at the recommended phase II dose.

Toxicity assessment. In the dose escalation phase, CRS was graded according to the Lee criteria ${ }^{66}$ and neurotoxicity-graded by the Common Terminology Criteria for Adverse Events v.4.0.3. Patients in the cohort expansion were graded according to the American Society for Transplantation and Cellular Therapy (ASTCT) criteria for CRS and ASTCT immune effector cell-associated neurotoxicity syndrome criteria for neurotoxicity ${ }^{67}$. For uniform reporting in the article, patients in the dose escalation group were regraded according to the ASTCT criteria. Adverse events were captured for all treated patients until disease relapse or death.

Response assessment. Response for patients with LBCL and patients with B-ALL with extramedullary disease without concurrent bone marrow or CNS involvement was assessed using the Lugano PET/CT criteria $^{67}$. For all other patients with B-ALL, CR was defined as $<5 \%$ bone marrow blasts by morphology. MRD negativity was defined as a bone marrow blast percentage $<10^{-4}$ by multiparameter flow cytometry.

Axi-cel patients. Consecutive patients with LBCL treated with standard-of-care axi-cel between 27 December 2017 and 9 April 2020 were identified. Patients were consented for collection of clinical data as well as blood and lymph node sampling on a clinical outcomes biorepository protocol. Patients with available tissue samples for IHC and/or quantitative flow cytometry were included in the analysis. The protocol was approved by the Stanford Internal Review Board (no. 43375). Clinical data were obtained retrospectively from the chart review. Treatment response was assessed radiographically according to the Lugano criteria.

CD19-22.BB.z production. CD19-22.BB.z products were manufactured in the automated closed-system CliniMACS Prodigy in a 7-11 d manufacturing process. All days provided in this CAR T production section are reflective of the manufacturing schema (Extended Data Fig. 2). The frozen patient apheresis was washed on the Lovo (Fresenius Kabi) and rested overnight in low-dose IL-2 before loading on the CliniMACS Prodigy. On day 0, the apheresis was enriched for CD4 and CD8 T cells before T cell activation with TransAct (Miltenyi Biotec). On day 1, $\mathrm{T}$ cells were transduced with CD19-22.BB.z lentiviral vector (Fig. 2a) at a multiplicity of infection of 40. TransAct was subsequently washed out on either day 3 (new matrix) or day 5 (old matrix), followed by a series of media exchanges. On days 7, 9 or 11, when target dose was achieved, the final product was collected, sampled for quality control testing and cryopreserved. The product release criteria are listed in Supplementary Table 1. Iterative improvements to the manufacturing process were implemented to shorten the vein-to-vein time during the course of the trial.

IHC. Tissue microarrays were constructed with duplicate $0.6-\mathrm{mm}$ cores of formalin-fixed paraffin-embedded tissue from diagnostic biopsies ${ }^{68}$. Additional whole-tissue sections were evaluated from cases when available.

Immunohistochemical studies were performed on 4-mm-thick sections of the formalin-fixed paraffin-embedded tissue in tissue microarray or whole-section form. Automated immunostaining was performed using the Leica BOND-III (Leica Biosystems). Slides were stained with antibodies against CD19 (clone BT51E; prediluted mouse monoclonal antibody; Leica Biosystems) and CD22 (clone FPC1; prediluted mouse monoclonal antibody; Leica Biosystems).

The intensity of staining ( 0 , negative; 1 , weak; 2 , moderate; 3 , strong) and percentage of tumor cells showing staining $(0-100 \%)$ were evaluated independently then jointly scored by 2 pathologists (S.Y. and Y.N.). An H-score of 0-300 was generated by multiplying the intensity of positivity by the percentage of tumor cells with staining.

Detection of CD19-22.BB.z cells. CD19-22.BB.z cells were detected using the CD19 anti-idiotype antibody developed at the MD Anderson Cancer Center ${ }^{69}$. The CD19 anti-idiotype antibody was conjugated to DyLight 650 (Thermo Fisher Scientific) using an antibody labeling kit and stored at $-80^{\circ} \mathrm{C}$ for downstream flow cytometry use.

Phenotyping of manufacturing samples at apheresis, enrichment and final product collection. This section describes the methods for the phenotyping shown in Fig. 2. All samples were washed in FACS buffer (1× PBS, 2\% FCS), stained for a minimum of $30 \mathrm{~min}$ at $4^{\circ} \mathrm{C}$ before additional washes and running flow cytometry on the MACSQuant Analyzer 10 (Miltenyi Biotec). The MACS Comp bead kit (catalog no. 130-097-900; Miltenyi Biotec) was used for compensation controls. The antibodies used for these experiments are listed in Supplementary Table 2.

Flow cytometry for phenotyping and exhaustion profiling. All samples were washed in FACS buffer ( $1 \times \mathrm{PBS}, 3 \% \mathrm{FCS})$, stained for a minimum of $30 \mathrm{~min}$ at $4{ }^{\circ} \mathrm{C}$ before additional washes and running flow cytometry. UltraComp eBeads (catalog no. 01-2222-41; Invitrogen) were used for compensation controls and stained with the respective antibody from the antibody index below. Samples were run on the LSRFortessa X-20 (BD Biosciences) and stained using the antibodies from antibody index shown below.

Exhaustion and $\mathrm{T}$ cell subset phenotyping panel. This section describes the methods for the intracellular cytokine panel in Figs. 2 and 4 and Extended Data Fig. 2. Samples were stained with a panel backbone (CD3, CD4, CD8, CAR, viability) first. Subsequently, samples were split into two for either phenotyping of T cell subsets (CD45RA, CD45RO, CCR7, CD62L, CD95) or exhaustion markers (CD39, LAG3, PD-1); all samples were prepared and washed as described above. Analysis for this and the previous two sections were performed in FlowJo v10.5.3 (FlowJo LLC)

NALM6 tumor line antigen density check by flow cytometry. This section describes the methods used for the intracellular cytokine panel in Fig. 4. All NALM6 lines were divided into two and stained with either CD19 or CD22 antibody and prepared and washed as described above. 
Coculture of CD19-22.BB.z/CD22.BB.z product with NALM6 target cells. This section describes the methods used for the intracellular cytokine panel in Fig. 4. Clinical CAR T products were thawed and rested overnight before incubation in coculture assays with the NALM6 lines described above. During this 6-7 h coculture at $37^{\circ} \mathrm{C}$, cells were coincubated with monensin (Thermo Fisher Scientific) and CD107a. A phorbol myristate acetate/ionomycin (Sigma-Aldrich) condition was included as a positive control, along with no monensin, no CD107a or no tumor cells as negative controls. After coculture, cells were washed with FACS buffer and resuspended in an extracellular antibody cocktail consisting of CD3, CD4, CD8, CD69, CAR, LIVE/DEAD and CD107a and incubated for $30 \mathrm{~min}$ at $4^{\circ} \mathrm{C}$. Cells were subsequently washed out with FACS buffer before intracellular cytokine staining using the Fixation/Permeabilization Solution Kit (BD Biosciences). The kit protocol was followed to fix and permeabilize CAR T products, followed by resuspension in an intracellular antibody cocktail consisting of IFN- $\gamma$ and TNF- $\alpha$ and incubated overnight at $4{ }^{\circ} \mathrm{C}$ Cells were washed the following morning in FACS buffer before proceeding with flow cytometry run on the LSRFortessa X-20. The full panel of antibodies is shown in Supplementary Table 3.

Real-time peripheral blood CAR T phenotyping assay. A high-dimensional immunophenotyping flow cytometry panel was designed for immune profiling of CAR T and B cells in real time on the LSR II (BD Biosciences) and analyzed in Cytobank. Peripheral blood mononuclear cells (PBMCs) were isolated from fresh whole blood by gradient centrifugation on Ficoll (Ficoll-Paque PLUS; Sigma-Aldrich). Between 2-5 million PBMCs were stained with fixable Live/Dead aqua (Invitrogen) amine-reactive viability. Cells were then preincubated with $\mathrm{Fc}$ block (TruStain FcX; BioLegend) for $5 \mathrm{~min}$ before staining at room temperature with the panel of antibodies listed in Supplementary Table 4. CD19-22.BB.z cells were used as the positive batch control for the daily staining experiments. At least $10^{6}$ cells were acquired unless restricted by the number of cells isolated from $8 \mathrm{ml}$ of whole blood. The assay limit of detection for CAR T cells was calculated as 1 in $10^{4}$ of total acquired PBMCs.

qPCR measurement of in vivo CD19-22.BB.z-CAR expansion. DNA was extracted from whole blood $\left(2 \times 10^{6}-5 \times 10^{6}\right.$ PBMCs) using the QIAamp DNA Mini Kit (catalog no. 51306; QIAGEN) at baseline and on days 7, 14, 21, 28, 90 and 180 postCAR infusion. CAR presence was measured by qPCR using the primer and probe sequences provided in Supplementary Table 5. For the standard curve, a custom Minigene plasmid (Integrated DNA Technologies) was designed containing a partial CD19-22.BB.z sequence and a partial albumin sequence, which served as a control for normalization. The standard curve contained a tenfold serial dilution of plasmid between $5 \times 10^{6}$ and $5 \times 10^{\circ}$ copies. Both plasmid and patient DNA from each time point were run in triplicate, with each reaction containing $5 \mu \mathrm{l}$ of DNA (50 ng total), $100 \mathrm{nM}$ of forward and reverse albumin primers (or $100 \mathrm{nM}$ of forward and $200 \mathrm{nM}$ reverse CD19-22.BB.z primers), a 150-nM probe suspended in $10 \mu \mathrm{l}$ of TaqMan Fast Universal PCR Master Mix (2×), No AmpErase UNG or equivalent (Thermo Fisher Scientific) and $5 \mu \mathrm{l}$ of TE buffer (catalog no. AM9935; Invitrogen). The Bio-Rad CFX96 Touch Real-Time PCR Detection System was used for qPCR with $20 \mu \mathrm{l}$ per reaction. The quality metrics of all qPCR standard curve results were $R^{2}>0.99,-3.38>$ slope $>-3.71$ and efficiency $>86 \%$.

IsoPlexis for 32-plex cytokines. CD19-22.BB.z or CD22.BB.z products were magnetically selected into CD4 or CD8 T cells using microbeads (Miltenyi Biotec). $\mathrm{CD} 4^{+}$and $\mathrm{CD}^{+}$populations were subsequently cocultured with NALM6-double knockout, NALM6-CD19 or NALM6-CD22 at a 1:2 ratio (T cell:tumor) for $20 \mathrm{~h}$. After incubation and a viability check, tumor cells were depleted from the coculture and the remaining T cells were stained with CD22-Fc-AF647 for CD1922.BB.z detection. Subsequently, 30,000 viable cells were loaded onto the 32-plex human polyfunctional strength single-cell IsoCode chips (IsoPlexis); duplicate chips were run when sample was available. Chips were loaded into the IsoLight machine for scanning and data were analyzed using the IsoSpeak software v.2.8.0.0 (IsoPlexis). Statistical significance between stimulation conditions for single-cell polyfunctionality, percentage and PSI was done by Mann-Whitney $U$-test.

Tumor-killing IncuCyte proliferation assay. The tumor-killing ability of bispecific CD19-22.BB.z and single CD19 and CD22 CAR T cells was assessed in a coculture assay with either NALM6-wild type, NALM6-CD19 knockoutor NALM6-CD22 knockout at an effector:target ratio of 3:1. CD19-22.BB.z cells, all from the same donor, were grown on the CliniMACS Prodigy and CD19 and CD22 CAR T cells were grown using small-scale plate-based processes. All NALM6 cell lines were green fluorescent protein (GFP)+ ; untransduced $\mathrm{T}$ cells were included as a mock condition. Tumor killing was measured as a decrease in GFP overtime using the IncuCyte System (Sartorius) and normalized.

Antigen density assessment. Specimens were processed within $24 \mathrm{~h}$ of collection $($ mean $\pm \mathrm{h})$, stained using the antibody combination listed in Supplementary Table 6 and analyzed on the FACSLyric system (BD Biosciences). The median fluorescence intensities of CD19, CD20 and CD22 were determined under saturating antibody conditions for antigen density measurements; the antibody bound per cell was calculated by calibration with Quantibrite phycoerythrin beads (BD Biosciences) and custom BD Biosciences quantitation beads for allophycocyanin and Brilliant Violet 421 (BD Biosciences) after controlling for the fluorophore to antibody ratio.

Measurement of cell-free tumor DNA. Tumor DNA was extracted from archival paraffin-embedded tissue; PCR amplification of the IgH-VDJ, IgH-DJ and Igא/Ig $\lambda$ regions using universal consensus primers was performed by NGS to determine the tumor clonotype(s) (Adaptive Biotechnologies) ${ }^{70}$. ctDNA was measured from plasma extracted from blood obtained in EDTA tubes at pre-, 0, 7, 14, 21, 56 and $90 \mathrm{~d}$ postCD19-22.BB.z-CAR infusion.

Statistical analysis. Descriptive statistics were enumerated by median and IQR for continuous variables and counts and percentages for categorical variables. Fisher's exact (independent) and McNemar's (dependent) tests evaluated the association between categorical variables and Spearman correlation was used for the association between two random continuous variables. Between-group comparisons for continuous variables were performed using either a Student's $t$-test or Wilcoxon signed-rank test. All tests were two-sided. To associate CD19 antigen density with risk of progression, Firth's penalized logistic regression was used due to the small sample size. Overall survival was defined as the time from infusion to death from any cause. PFS was defined as the time from infusion to either disease progression or death from any cause. Patients were censored at the time of the last follow-up. Overall survival and PFS were estimated by Kaplan-Meier method. Response rates were summarized along with $95 \%$ Clopper-Pearson CIs. Analyses were exploratory and not adjusted for multiple comparisons. The statistical software packages used include R v.3.6.2 and Prism 8 (GraphPad Software). $P$ values generated in Prism 8 did not go below $P<0.0001$.

Reporting Summary. Further information on research design is available in the Nature Research Reporting Summary linked to this article.

\section{Data availability}

All requests for raw and analyzed data will be reviewed by the corresponding author to determine if the request if subject to any intellectual property or confidentiality considerations. Patient-related data not included in the paper were generated as part of clinical trials and may be subject to patient confidentiality. Any data and materials that can be shared will be released via a material transfer agreement. The source data for Figs. 1-4 and Extended Data Figs. 1-7 are provided with the paper. The CD19-22 bispecific CAR sequence is in the patent application (U.S. Provisional Patent Application no. 62/135442) filed on 19 March 2015, titled Dual Specific Anti-Cd22-Anti-Cd19 Chimeric Antigen Receptors; the amino acid sequence is provided in Supplementary Fig. 2 .

\section{References}

66. Lee, D. W. et al. Current concepts in the diagnosis and management of cytokine release syndrome. Blood 124, 188-195 (2014).

67. Lee, D. W. et al. ASTCT consensus grading for cytokine release syndrome and neurologic toxicity associated with immune effector cells. Biol. Blood Marrow Transplant. 25, 625-638 (2019).

68. Marinelli, R. J. et al. The Stanford Tissue Microarray Database. Nucleic Acids Res. 36, D871-D877 (2008).

69. Jena, B. et al. Chimeric antigen receptor (CAR)-specific monoclonal antibody to detect CD19-specific T cells in clinical trials. PLoS ONE 8, e57838 (2013).

70. Ching, T. et al. Analytical evaluation of the clonoSEQ Assay for establishing measurable (minimal) residual disease in acute lymphoblastic leukemia, chronic lymphocytic leukemia, and multiple myeloma. BMC Cancer 20, 612 (2020).

\section{Acknowledgements}

This work was supported by the California Institute for Regenerative Medicine (award no. CLIN2-10846 to C.L.M., principal investigator) and from the National Cancer Institute (grant no. 5P30CA124435 to C.L.M., grant no. U54 CA232568-01 to C.L.M and grant no. 2P01CA049605-29A1 to C.L.M. and D.M.). C.L.M. is a member of the Parker Institute for Cancer Immunotherapy, which supports the Stanford University Cancer Immunotherapy Program. The work was also supported by the Virginia and D.K. Ludwig Fund for Cancer Research (C.L.M.). This work received testing support from the National Gene Vector Biorepository, which is funded by the National Heart, Lung, and Blood Institute, National Institutes of Health (NIH), contract no. 75N92019D00018. J.Y.S. received support from a 2019-2020 American Society of Hematology Research Training Award for Fellows. K.L.D. is supported by the Maternal and Child Health Research Institute as the Anne T. and Robert M. Bass Endowed Faculty Scholar in Pediatric Cancer and Blood Diseases. R.G.M. is the Taube Distinguished Scholar for Pediatric Immunotherapy at Stanford University School of Medicine. We thank our contract manufacturing organizations: the Laboratory of Cell and Gene Medicine, Stanford University and Miltenyi Biotec. We thank Adaptive Biotechnologies for their collaboration in measuring cell-free MRD and Becton Dickinson for assistance in developing the antigen quantitation assays. We thank IsoPlexis for their collaboration in 
performing the single-cell cytokine analysis on manufactured CAR T products in Fig. $4 \mathrm{j}$. Figs. 2 and 4 and Extended Data Fig. 1 were created using BioRender.com.

\section{Author contributions}

C.L.M. and D.B.M. conceived and designed the study. D.B.M., S.F., J.Y.S., S.P., L.M., J.H.B., N.M.H., J.O., B.S., J.C., M.I., S.Y., M.G.O., E.Y., Z.E., W.R., R. Lynn and H.C. collected and assembled the data. C.L.M., D.B.M., S.F., J.Y.S., S.P., L.M., J.H.B., M.F., B.S., S.Y., Y.N., J.T., I.K., C.M. and A.J. analyzed and interpreted the data. C.L.M., D.B.M., S.F., J.Y.S., S.P., L.M., J.H.B., N.M.H., J.O., M.F., P.S., B.S., R.G.M., S.A., L.J., R. Lowsky, E.M., R.S.N., A.R.R., S.S., J.S., W.K.W., L.S., S.R., K.L.D., K.A.K., N.N.S., H.Q. and T.F. wrote the manuscript

\section{Competing interests}

C.L.M. is an inventor on a patent application for CD19/22-CAR T cells and holds several patent applications in the area of CAR T cell immunotherapy. C.L.M. is a founder of, holds equity in and receives consulting fees from Lyell Immunopharma and Syncopation Life Sciences. She has also received consulting fees from NeoImmune Tech, Nektar Therapeutics, Immatics, GlaxoSmithKline and Apricity Health and royalties from Juno Therapeutics for the CD22-CAR. She holds equity in Vor Biopharma and Apricity Health. D.B.M. has consulted for Kite-Gilead, Juno Therapeutics-Celgene-Bristol Myers Squibb, Novartis and Adaptive Biotechnologies. He has received research for Kite-Gilead and Adaptive Biotechnologies. S.F. has consulted for Lonza PerMed, Gradalis, Obsidian and Samsara Biocapital. L.M. has consulted for Amgen, Pfizer and Kite-Gilead. She has received research funding from Adaptive Biotechnologies, Astellas Pharma, Servier and Baxalta. S.P. has consulted for Cellares. R.S.N. has consulted for Kuur Therapeutics, who are developing CAR invariant NKT cells, and CoImmune, who are developing CAR cytokine-induced killer cells. A.R.R. has received research support from Pharmacyclics and performed a one-time ad hoc scientific advisory board role for Nohla and Koledio. $\mathrm{He}$ is a medical expert witness for the U.S. Department of Justice; his brother works for Johnson \& Johnson. H.Q. is an inventor on a patent application for CD19/22-CAR T cells and holds several patent applications in the area of CAR T cell immunotherapy. She has also received royalties from Lentigen via the NIH for the thymic stromal lymphopoietin receptor-CARs. I.K., C.M. and A.J. are full-time employees and shareholders of Adaptive Biotechnologies. R.G.M. holds several patent applications in the area of CAR T cell immunotherapy and is a consultant for Lyell Immunopharma, Xyphos Biosciences, GammaDelta Therapeutics, Zai Lab and Aptorum Group. The other authors declare no conflicts of interest.

\section{Additional information}

Extended data is available for this paper at https://doi.org/10.1038/s41591-021-01436-0. Supplementary information The online version contains supplementary material available at https://doi.org/10.1038/s41591-021-01436-0.

Correspondence and requests for materials should be addressed to C.L.M. or D.B.M.

Peer review information Nature Medicine thanks Katayoun Rezvani, Saar Gill and the other, anonymous, reviewer(s) for their contribution to the peer review of this work. Saheli Sadanand was the primary editor on this article and managed its editorial process and peer review in collaboration with the rest of the editorial team.

Reprints and permissions information is available at www.nature.com/reprints. 


\begin{tabular}{cc}
\hline $\begin{array}{c}\text { Pre axi-cel CD19 } \\
\text { Molecules/Cell }\end{array}$ & $\begin{array}{c}\text { Probability of } \\
\text { Relapse }\end{array}$ \\
\hline 100 & $99.80 \%$ \\
\hline 1000 & $87.80 \%$ \\
1505 & $77.30 \%$ \\
\hline 2007 & $66.70 \%$ \\
\hline 2512 & $57.00 \%$ \\
\hline 2934 & $49.90 \%$ \\
\hline 3508 & $41.80 \%$ \\
\hline 4004 & $36.00 \%$ \\
\hline 4519 & $31.00 \%$ \\
\hline 5012 & $27.10 \%$ \\
\hline 5495 & $23.90 \%$ \\
\hline 6026 & $20.90 \%$ \\
\hline 6531 & $18.60 \%$ \\
\hline 7039 & $16.60 \%$ \\
7499 & $15.00 \%$ \\
\hline 10000 & $9.40 \%$ \\
\hline 31623 & $1.20 \%$ \\
\hline 100000 & $0.2 \%$ \\
\hline
\end{tabular}

Extended Data Fig. 1 | Model-based prediction of relapse after axi-cel. Estimated risk of relapse for a given pre-treatment CD19 antigen density based on a fitted penalized logistic regression model. 


\begin{tabular}{ccc}
\hline Pt ID & CD19 & CD19 H-score \\
\hline A181 & 46805 & 300 \\
A182 & 19994 & N/A \\
\hline A152 & 15236 & 285 \\
\hline A129 & 9512 & 300 \\
\hline A150 & 7569 & 150 \\
\hline A161 & 6867 & 285 \\
\hline A116 & 6539 & 180 \\
\hline A110 & 6396 & 300 \\
\hline A123 & 5810 & 285 \\
\hline A125 & 5430 & N/A \\
\hline A154 & 4371 & 300 \\
\hline A122 & 2924 & 285 \\
\hline A81 & 2685 & 240 \\
\hline A139 & 2327 & N/A \\
\hline A140 & 952 & 200 \\
\hline
\end{tabular}

Extended Data Fig. 2 | Evaluation of pre-axi-cel antigen density. CD19 IHC H-score and antigen density by quantitative flow cytometry in 15 patients prior to axi-cel. Spearman correlation for these values shown in $1 \mathrm{G}$. 


\section{CD19-22 CONSORT DIAGRAM}

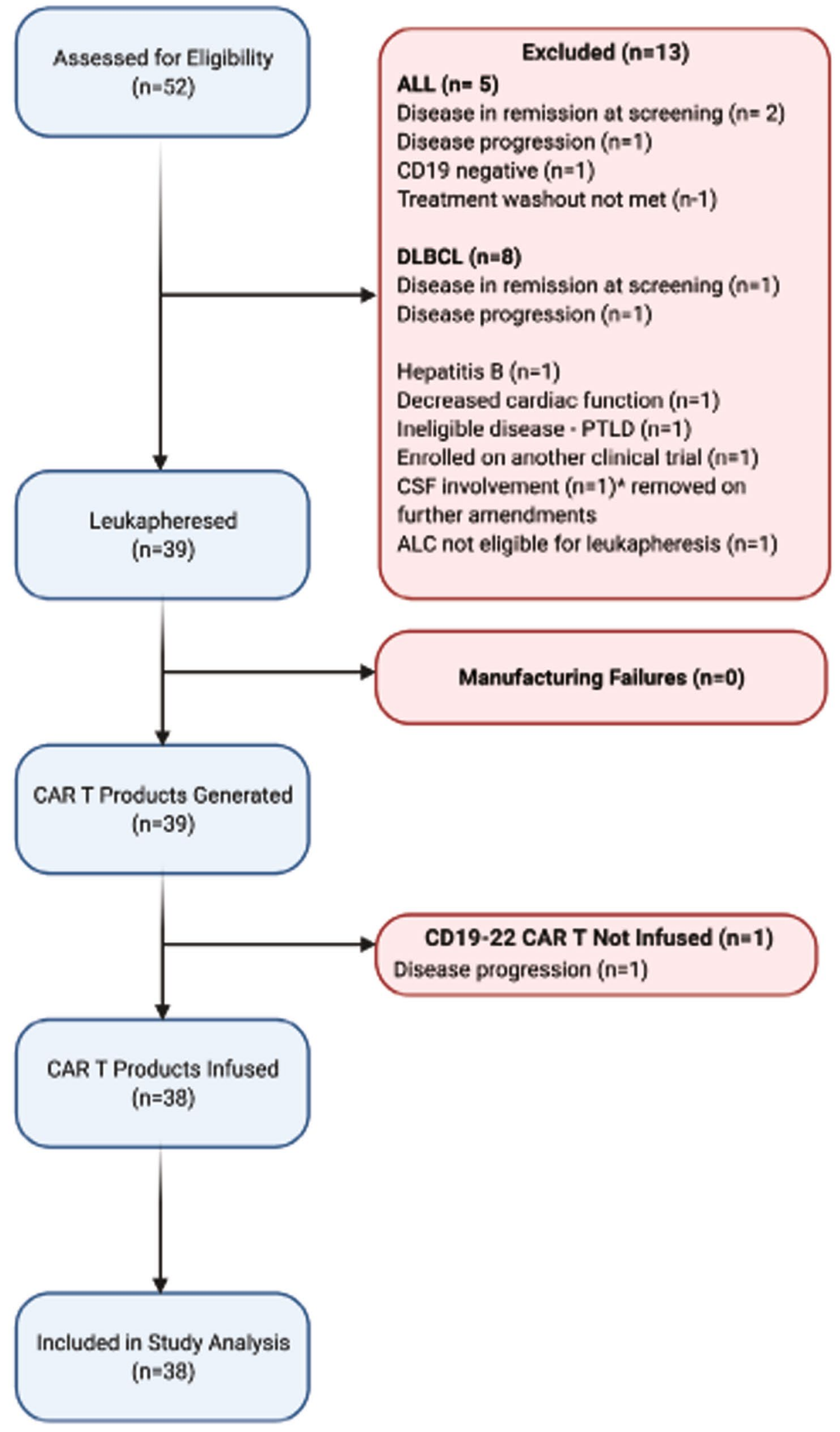

Extended Data Fig. 3 | CONSORT Diagram. Consort diagram of CD19-22-CD8.BB.z clinical trial. 
A.

TransAct Washout/Media Exchange

Old Manufacturing Matrix

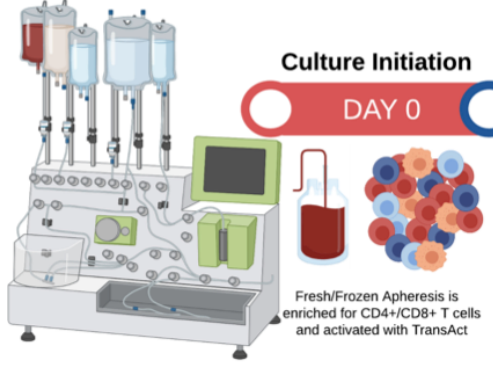

Culture Initiation Transduction

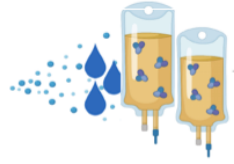

Culture Check Harvest

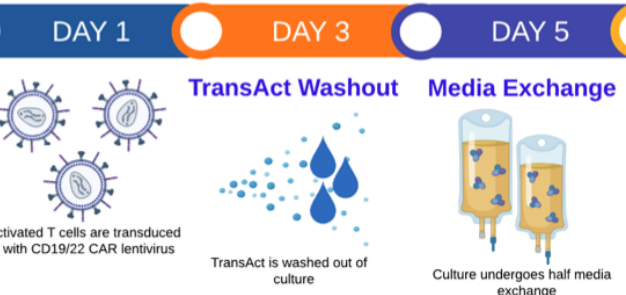

DAY $6 \bigcirc \operatorname{DAY} 7$

New Manufacturing Matrix

B.

\begin{tabular}{|c|c|c|c|c|}
\hline \multicolumn{6}{|c|}{ CD19/22 CAR T Product Characteristics } \\
\hline Culture Days (CD) & $\mathbf{7}$ & $\mathbf{9}$ & $\mathbf{1 1}$ & Total \\
\hline \# Products & 32 & 5 & 2 & 39 \\
\hline Old Matrix & 4 & 5 & 0 & 9 \\
\hline New Matrix & 28 & 0 & 2 & 30 \\
\hline Dose Level 1 & 5 & 3 & 0 & 8 \\
\hline Dose Level 2 & 27 & 2 & 2 & 31 \\
\hline (CD) Mean Doubling Time & (7) 1.92 & (9) 2.10 & (11) 2.91 & 1.99 \\
\hline
\end{tabular}

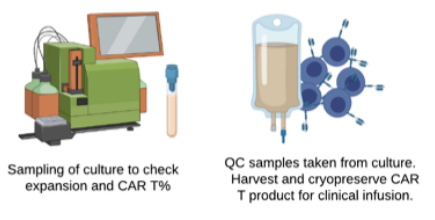

C.

\begin{tabular}{|c|c|c|c|}
\hline Cohort & DLBCL & ALL & Total \\
\hline \# Products & 22 & 17 & 39 \\
\hline Mean CAR T\% & $57.70 \%$ & $63.10 \%$ & $59.58 \%$ \\
\hline Mean VCN & 2.06 & 2.45 & 2.23 \\
\hline
\end{tabular}

D.

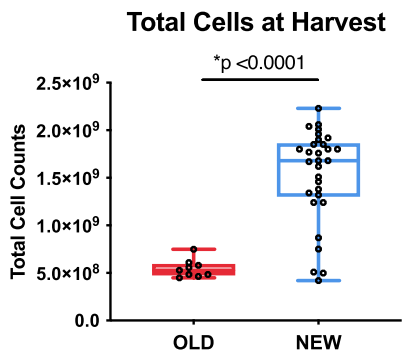

E.

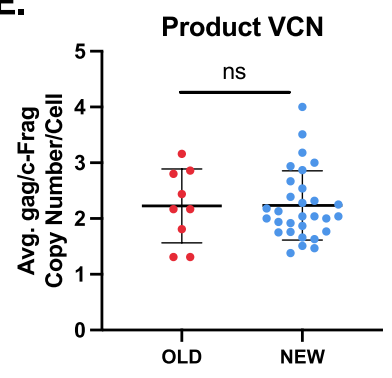

F.

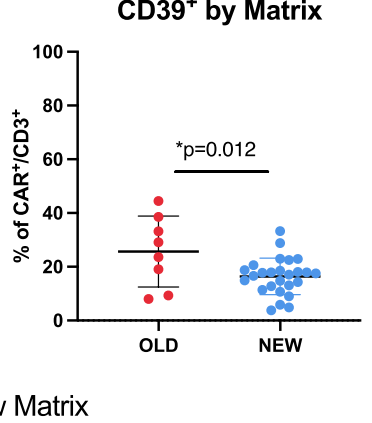

G.

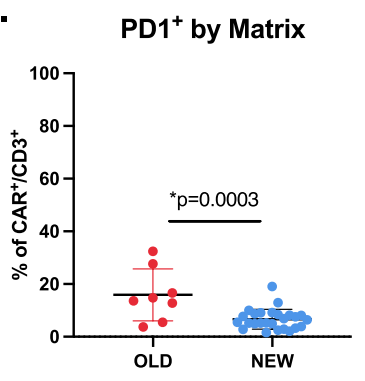

Old Matrix New Matrix

H.
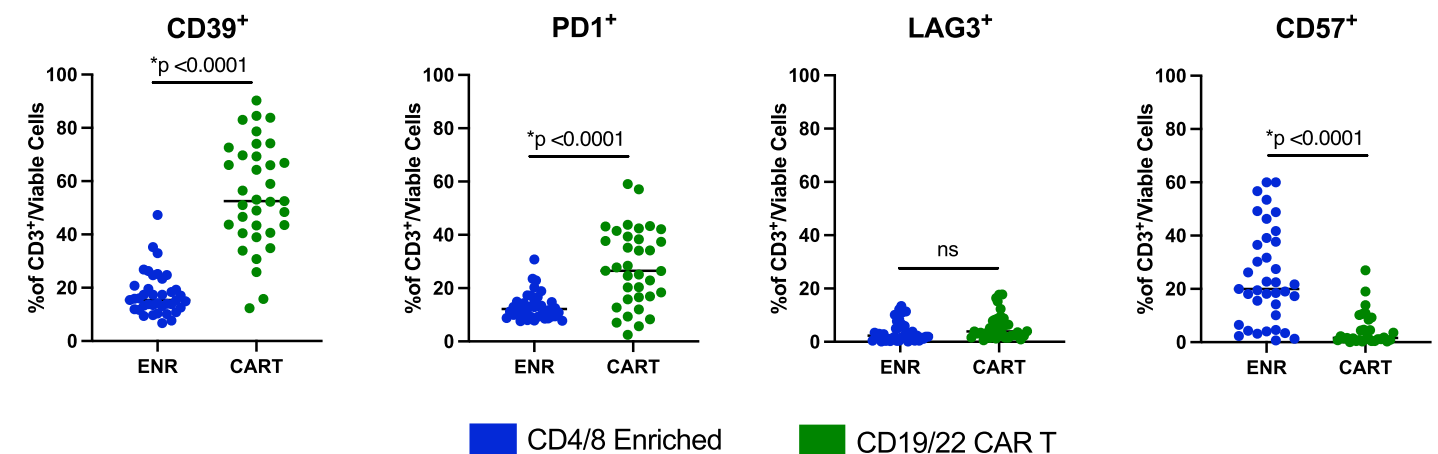

Extended Data Fig. 4 | CD19/22 CAR T manufacturing flowchart and product breakdown by culture days, manufacturing matrix, dose level, and disease. a, Manufacturing schematic detailing difference in timing of TransAct washout between the Old and New Matrix. b, Table of Infusion Product Characteristics, categorized by the number of culture days. c, Table of Infusion Product Characteristics, categorized by disease cohort. $\mathbf{d}$, Significant difference in product expansion between the old and new matrix at harvest $(p<0.0001$, $t$-test, two tailed). Box plot center line corresponds to the median; hinges correspond to the $25^{\text {th }}$ and $75^{\text {th }}$ percentiles with the whiskers signifying minimum and maximum values e, No significant difference in vector copy number based on manufacturing matrix. $\mathbf{f}, \mathbf{g}$, Significant decreases in CD39+ $(p=0.012$, t-test, two-tailed $)$ and PD1+ ( $p=0.0003$, t-test, two-tailed) CAR T cells manufactured using the new matrix versus the old matrix. $\mathbf{h}$, Significant increases in CD39+ and PD1+ and a significant decrease in CD57+ expression on CD3+ cells from CD4/8 enriched to final CAR T product ( $p<0.0001$, $t$-test, two-tailed; for all 3 tests). No difference in LAG3 expression. 


\begin{tabular}{|c|c|c|c|}
\hline Adverse Event & Grade 3 & Grade 4 & All Grades \\
\hline \multicolumn{4}{|c|}{ Blood and Lymphatic System Disorders } \\
\hline Anemia & $34 \%$ & $0 \%$ & $95 \%$ \\
\hline Decreased neutrophil count & $29 \%$ & $34 \%$ & $89 \%$ \\
\hline Decreased Platelet Count & $26 \%$ & $24 \%$ & $87 \%$ \\
\hline Eosinophilia & $3 \%$ & $0 \%$ & $3 \%$ \\
\hline \multicolumn{4}{|l|}{ Cytokine Release Syndrome } \\
\hline CRS & $3 \%$ & $3 \%$ & $76 \%$ \\
\hline Fever & $8 \%$ & $0 \%$ & $76 \%$ \\
\hline Hypotension & $8 \%$ & $0 \%$ & $55 \%$ \\
\hline Tachycardia & $0 \%$ & $0 \%$ & $29 \%$ \\
\hline Hypoxia & $3 \%$ & $3 \%$ & $13 \%$ \\
\hline Transaminitis & $0 \%$ & $5 \%$ & $11 \%$ \\
\hline Artrial Fibrillation & $3 \%$ & $0 \%$ & $5 \%$ \\
\hline \multicolumn{4}{|l|}{ Neurotoxicity } \\
\hline CRES & $8 \%$ & $3 \%$ & $37 \%$ \\
\hline Altered Mental Status & $5 \%$ & $0 \%$ & $29 \%$ \\
\hline Tremor & $3 \%$ & $0 \%$ & $8 \%$ \\
\hline Cerebral edema & $3 \%$ & $0 \%$ & $3 \%$ \\
\hline Seizure activity & $0 \%$ & $0 \%$ & $3 \%$ \\
\hline Dysphasia & $3 \%$ & $0 \%$ & $3 \%$ \\
\hline \multicolumn{4}{|l|}{ Infections } \\
\hline URI & $0 \%$ & $0 \%$ & $29 \%$ \\
\hline Lung Infection & $3 \%$ & $0 \%$ & $3 \%$ \\
\hline Skin Infection & $3 \%$ & $0 \%$ & $3 \%$ \\
\hline Sepsis & $0 \%$ & $3 \%$ & $3 \%$ \\
\hline \multicolumn{4}{|l|}{ Immune System Disorder } \\
\hline Macrophage Activation Syndrome & $5 \%$ & $3 \%$ & $8 \%$ \\
\hline \multicolumn{4}{|l|}{ Gastrointestinal Disorders } \\
\hline Anorexia & $8 \%$ & $0 \%$ & $37 \%$ \\
\hline Nausea & $0 \%$ & $0 \%$ & $26 \%$ \\
\hline Constipation & $0 \%$ & $0 \%$ & $11 \%$ \\
\hline Abdominal Bloating & $3 \%$ & $0 \%$ & $5 \%$ \\
\hline ascites & $3 \%$ & $0 \%$ & $3 \%$ \\
\hline \multicolumn{4}{|l|}{ General Disorders } \\
\hline Fatigue & $3 \%$ & $0 \%$ & $58 \%$ \\
\hline Headache & $0 \%$ & $0 \%$ & $26 \%$ \\
\hline Dizziness & $0 \%$ & $0 \%$ & $13 \%$ \\
\hline Acute kidney injury & $3 \%$ & $0 \%$ & $5 \%$ \\
\hline Infusion related reaction & $3 \%$ & $0 \%$ & $3 \%$ \\
\hline \multicolumn{4}{|l|}{ Musculoskeletal Disorders } \\
\hline Myalgia & $0 \%$ & $0 \%$ & $24 \%$ \\
\hline Arthralgia & $0 \%$ & $0 \%$ & $13 \%$ \\
\hline Generalized muscle weakness & $3 \%$ & $0 \%$ & $3 \%$ \\
\hline \multicolumn{4}{|l|}{ Cardiovascular Disorders } \\
\hline Hypertension & $3 \%$ & $0 \%$ & $5 \%$ \\
\hline Respiratory Failure & $0 \%$ & $3 \%$ & $3 \%$ \\
\hline
\end{tabular}

Extended Data Fig. 5 | Adverse events summary. Adverse events with a Grade $\geq 3$ incidence rate of $5 \%$ or higher were included. CAR specific toxicities were included regardless of incidence. 


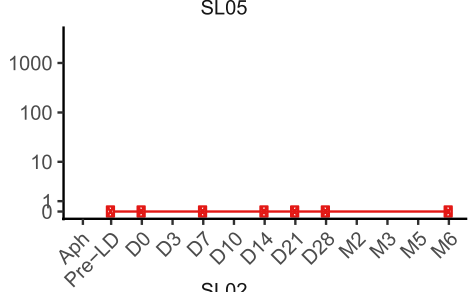

SL25
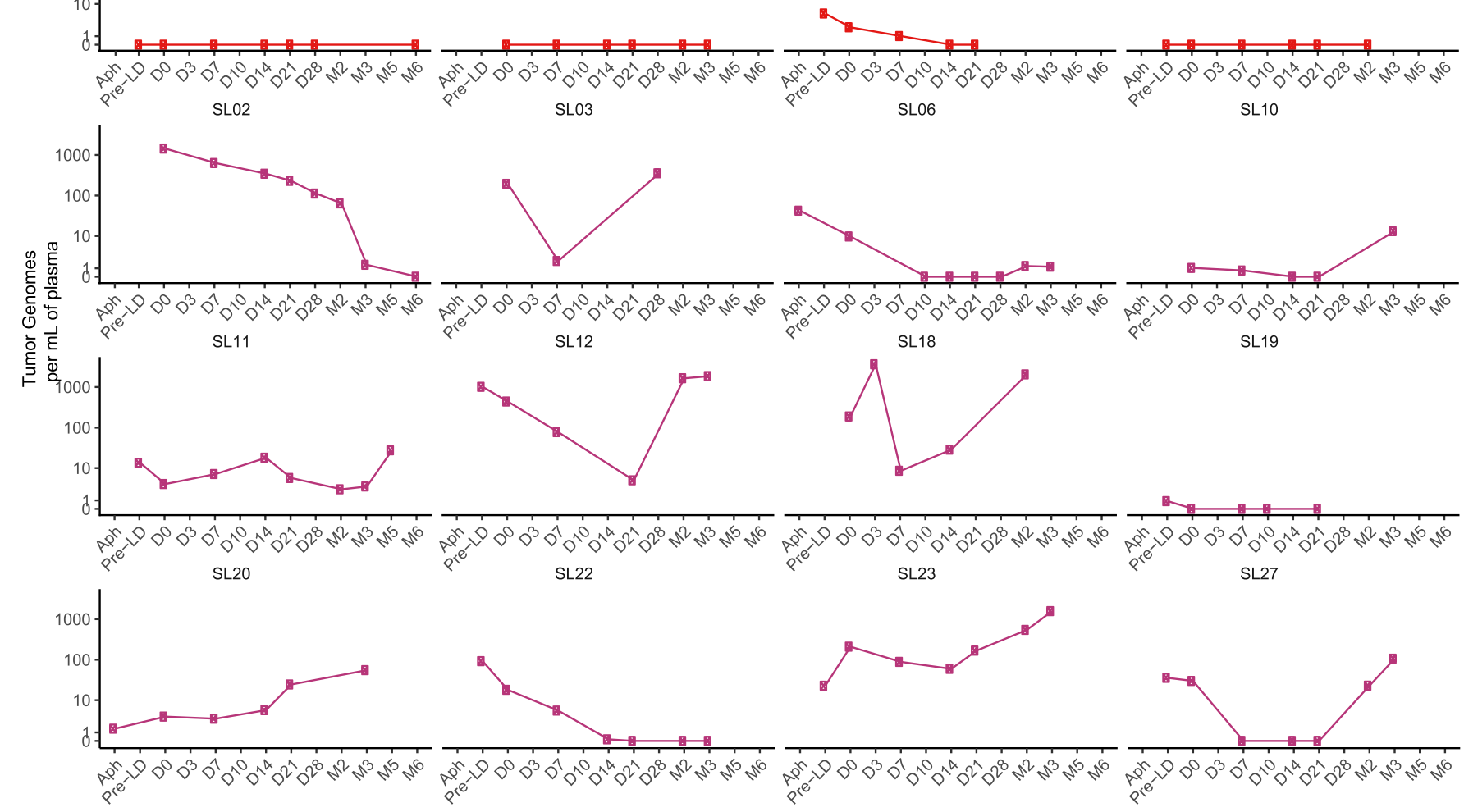

Timepoint

Extended Data Fig. 6 | LBCL disease monitoring by ctDNA. Patients with baseline tumor sample were assessed for a dominant clone to allow for disease tracking by cell free tumor DNA in the peripheral blood. Measurements were performed at pre-specified timepoints from pre-infusion to the time of disease progression. 
CD19-22.BBz B-ALL Disease Kinetics
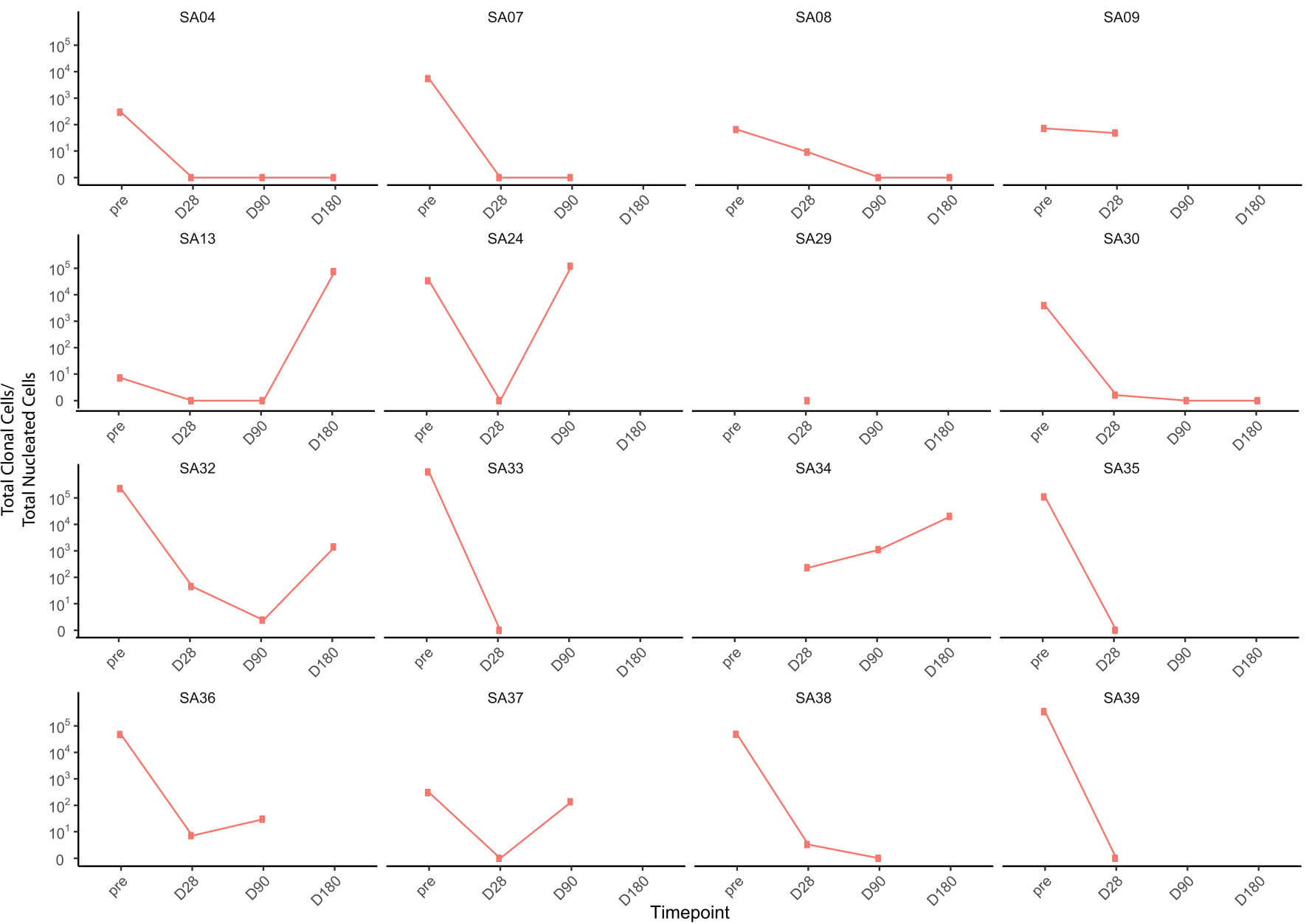

SA39

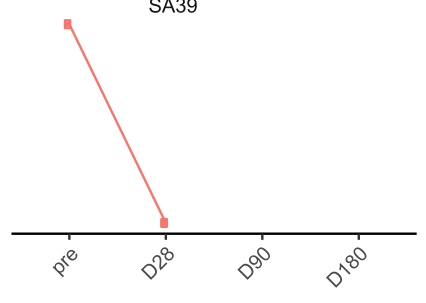

Extended Data Fig. 7 | B-ALL disease monitoring by ClonoSeq. Patients with baseline tumor sample were assessed for a dominant clone to allow for disease tracking by cell free tumor DNA in the peripheral blood. Measurements were performed at pre-specified timepoints from pre-infusion to the time of disease progression. 
A.

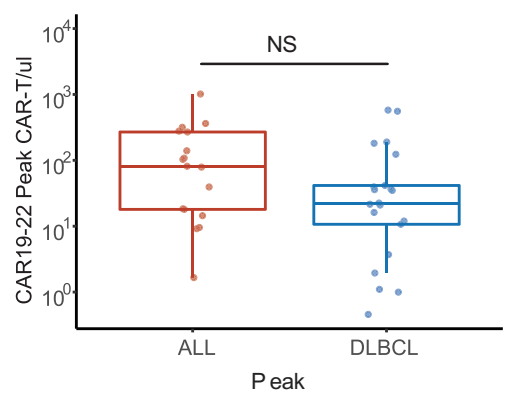

c.

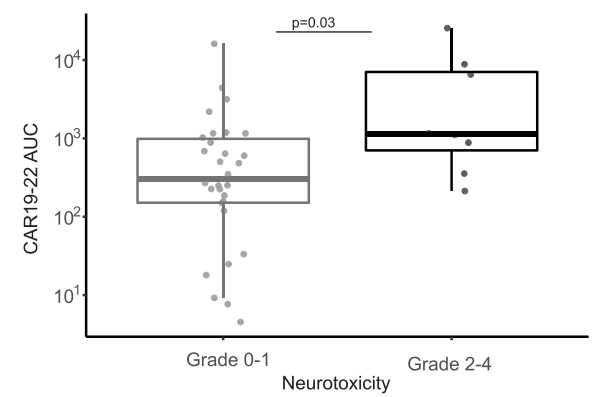

E.

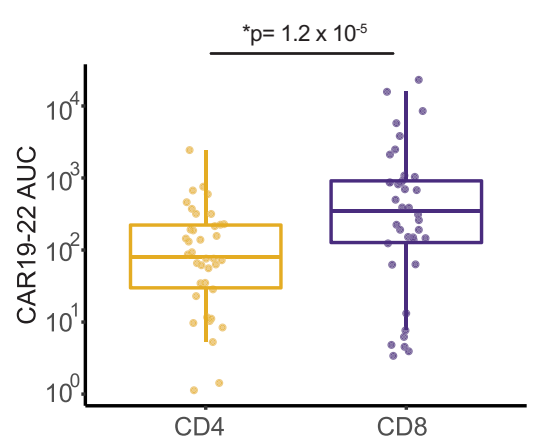

G.

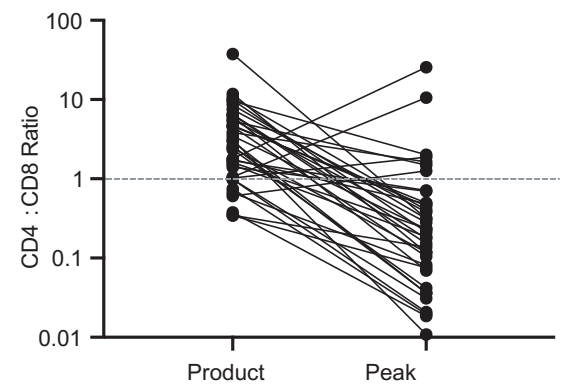

H .

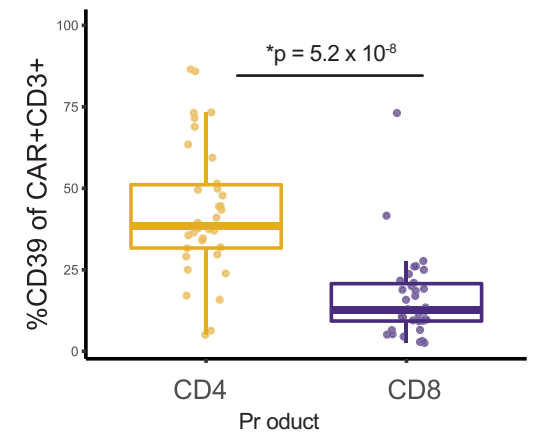

I.
B

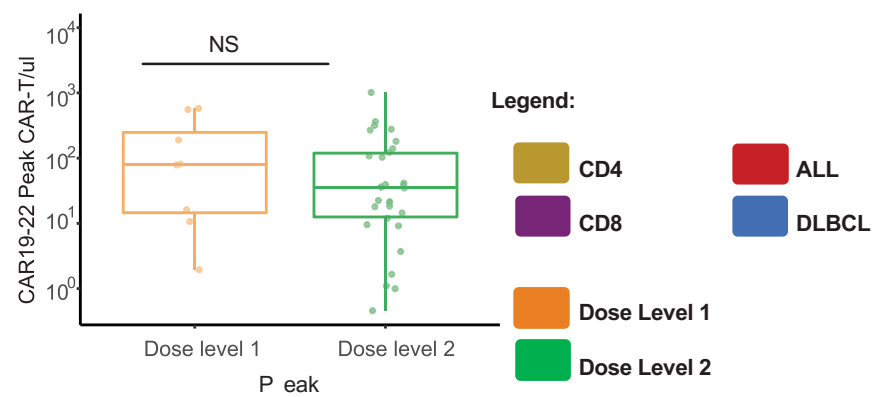

D.

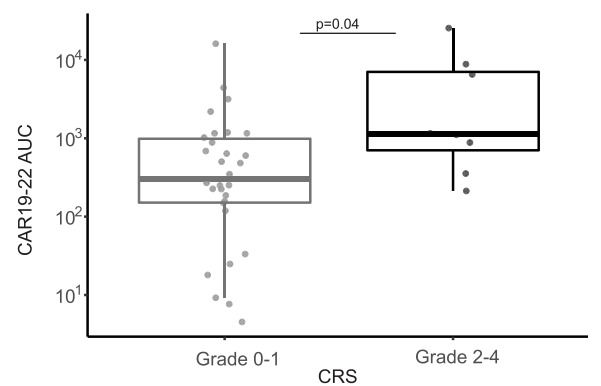

F.

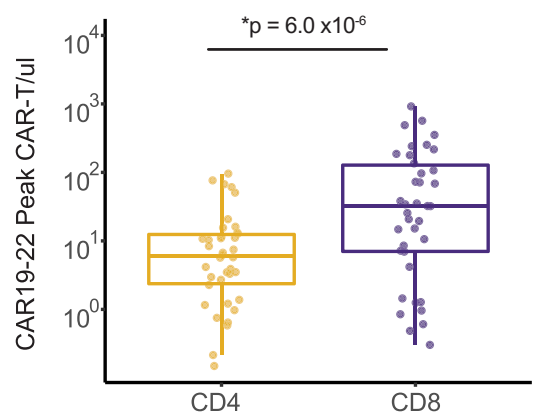

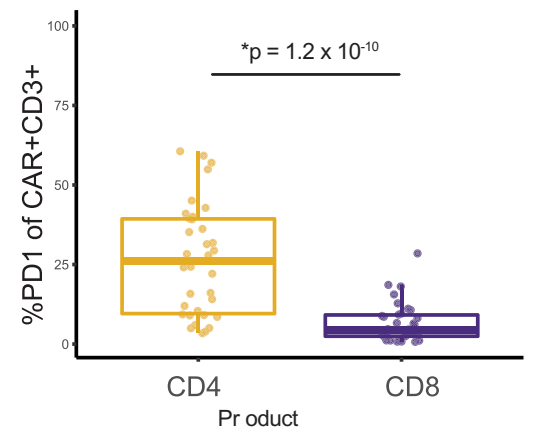

Extended Data Fig. 8 | See next page for caption. 


\section{NATURE MEDICINE}

Extended Data Fig. 8 | In vivo CAR-T cell expansion is CD8 predominant and relates to exhaustion phenotype of CAR-T products. a,b Peak CD19-22. BBZ cells as measured by flow cytometry compared by (a) disease type and (b) dose level. No significant difference observed. c,d. CD19-22.BB.z AUC was measured by the trapezoidal method from infusion until Day 60 post infusion.Grades 2-4 (d) CRS $(p=0.04)$ and $(\mathbf{c})$ neurotoxicity $(p=0.03)$ associated with higher CD19-22.BBZ AUC. Comparisons by Wilcoxon rank sum. e, Area under the curve calculated by trapezoidal integration of CD4 and CD8 CD1922.BB.z cells from infusion to 2 months post infusion. The AUC for CD8 was greater than that of $C D 4\left(p=1.2 \times 10^{-5}\right)$ f, Peak expansion of CD4 and CD8 CD19-22.BB.z-CAR T cells measured by flow cytometry. Peak CD8 CD19-22.BB.z-CAR was greater than that of CD4 $\left(p=6.0 \times 10^{-6}\right)$. Comparison in e and $\mathbf{f}$ performed by paired Wilcoxon signed rank test. $\mathbf{g}$, Change in the CD4:CD8 ratio from CD19-22.BB.z infusion product to peak expansion, shows the outgrowth of CD4 cells during manufacturing is not seen in the patients at time of peak CAR expansion, where CD8 CAR T+ cells are more abundant. $N=38$ patients for a-g. $\mathbf{h}, \mathbf{i}$ Significantly higher percentage of CD4 CAR T cells in the infusion product express CD39 $\left(p=5.2 \times 10^{-8}\right)$ and PD-1 $(p=1.2$ $\times 10^{-10}$ ) compared to CD8 CAR-T cells (Wilcoxon signed rank) $(n=34$ patients). Gating on CD3+CAR+ cells. Box plot center line corresponds to the median; hinges correspond to the $25^{\text {th }}$ and $75^{\text {th }}$ percentiles with the whiskers extending to the smallest or largest value at most $1.5 \times$ IQR from the hinge. All tests were two-sided and not adjusted for multiple comparisons. 
A.

DLBCL: CD19 by IHC

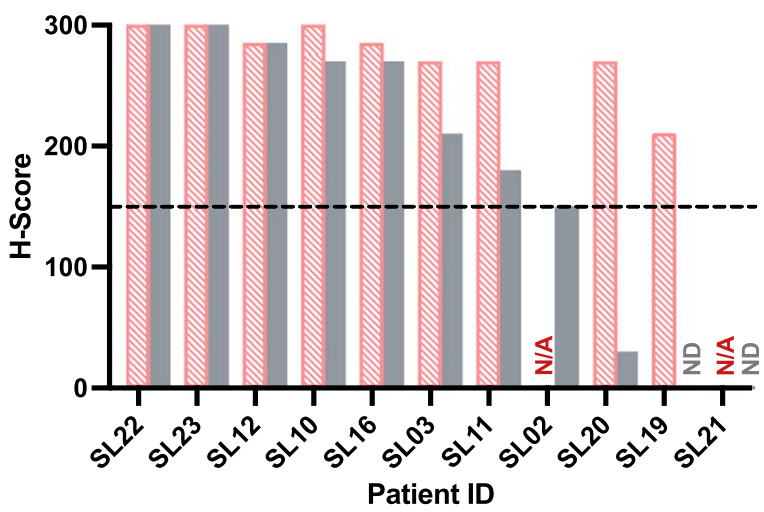

B. DLBCL: CD22 by IHC

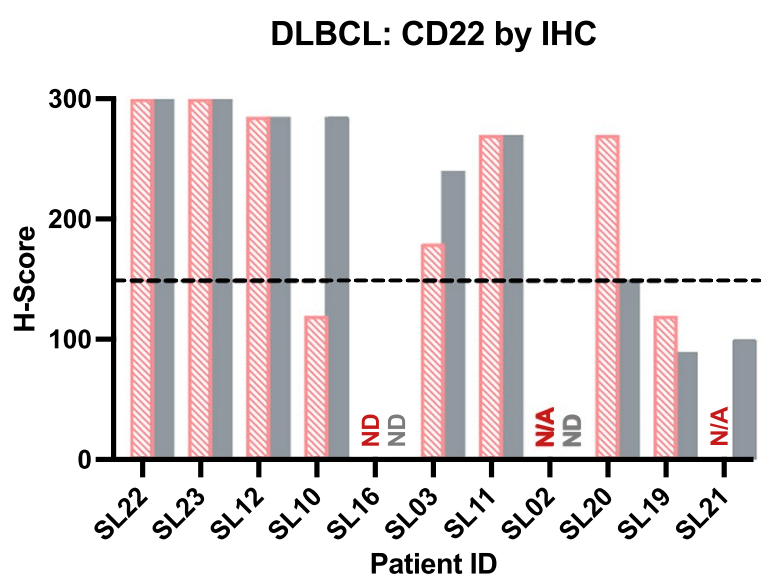

Pre-CAR 19-22

N/A - No data point
Post Progression

ND - Not Detectable

Extended Data Fig. 9 | Site density change after CD19-22.BB.z. a, Waterfall plot of assessment of CD19 for patients with LBCL by IHC H-score pre-CD19-22.BB.z and post progression. Cutoff for CD19 positivity was 150. b. Waterfall plot of assessment of CD22 in patients with LBCL by IHC H-score pre-CD19-22-CD8.BB.z and post progression ( $n=11$ patients for $a-b)$. Cutoff for CD22 positivity was 150 . 
A.

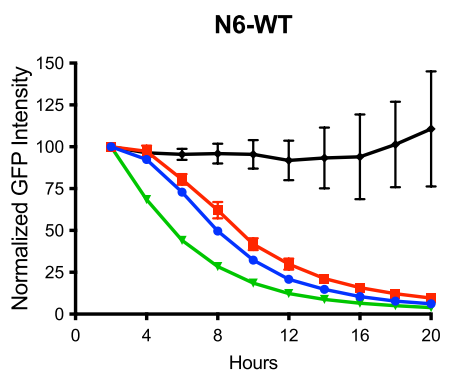

N6-CD22 (CD19KO)

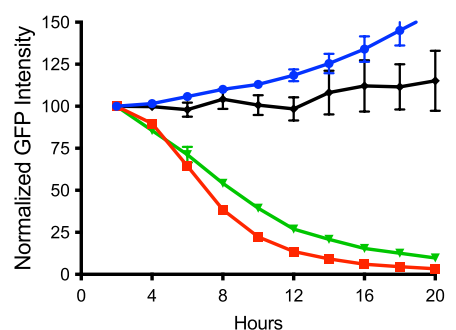

N6-CD19 (CD22KO)

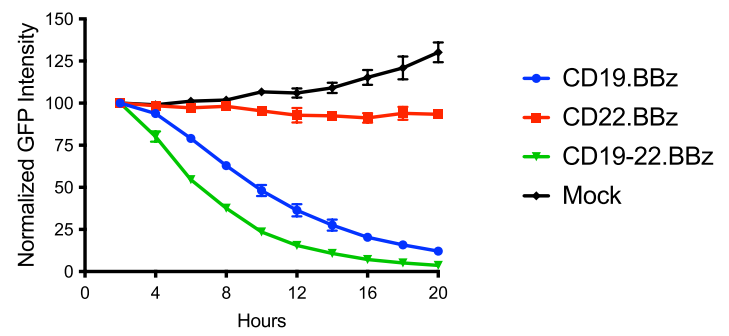

B.

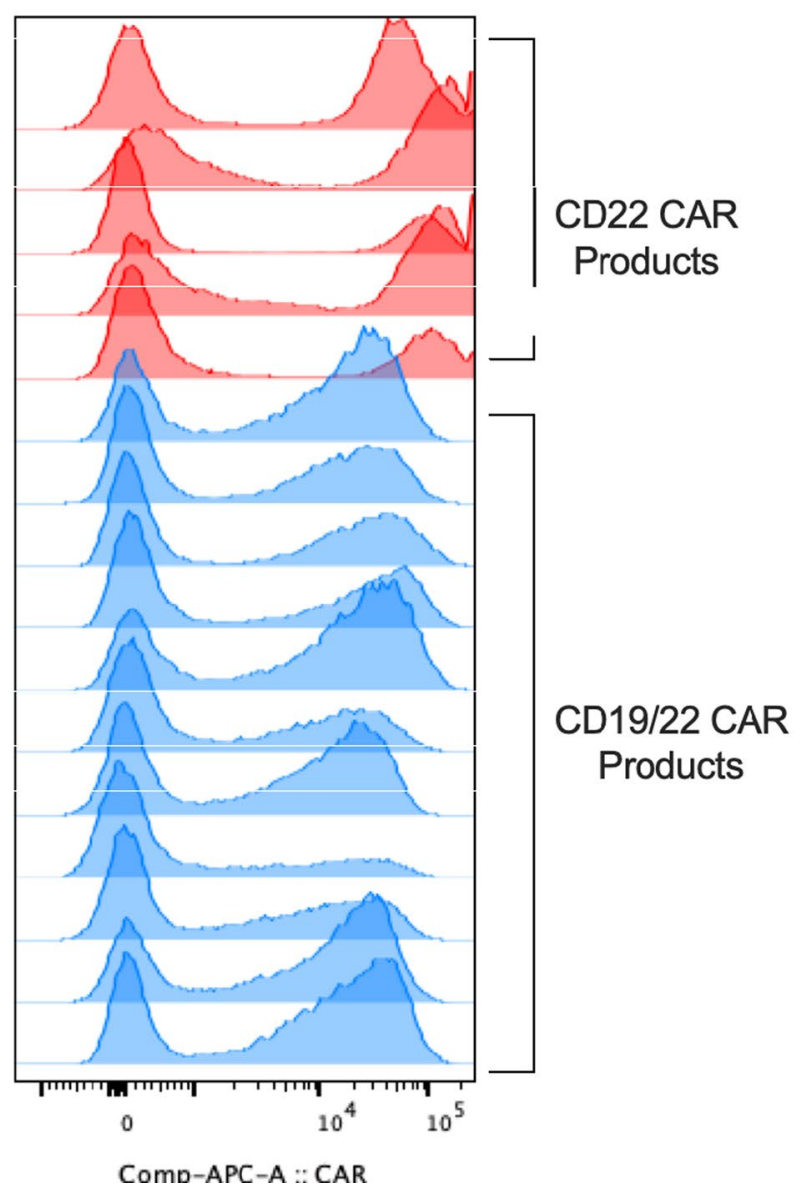

C. \begin{tabular}{|l|l|l|l|l}
\hline Pt \#AR T & VCN \\
\hline
\end{tabular} CD22 Products

\begin{tabular}{|r|r|r|}
\hline Pt 09 & 59.5 & 0.37 \\
\hline Pt 07 & 65.7 & 1.02 \\
\hline Pt 17 & 33.8 & 0.38 \\
\hline Pt 14 & 64.8 & 1.01 \\
\hline Pt 11 & 38.4 & 0.76 \\
\hline \multicolumn{2}{|c|}{ CD19/22 Products } \\
\hline Pt 18 & 71.2 & 2.25 \\
\hline Pt 10 & 54.6 & 1.77 \\
\hline Pt 11 & 52.9 & 1.75 \\
\hline Pt 15 & 52.3 & 1.87 \\
\hline Pt 16 & 73.1 & 2.54 \\
\hline Pt 23 & 47.8 & 1.94 \\
\hline Pt 25 & 63.3 & 2.13 \\
\hline Pt 28 & 31 & 1.92 \\
\hline Pt 34 & 49.9 & 2.04 \\
\hline Pt 38 & 71.2 & 3 \\
\hline Pt 39 & 70.9 & 2.32 \\
\hline
\end{tabular}

Extended Data Fig. 10 | Functional assessment of CD19-22 scFvs by Incucyte and comparison of MFI, transduction efficiency and VCN of CD19-22. BB.z-CAR and CD22-BB.z.CAR. a, Incucyte shows tumor killing after co-culture with CD19-22, CD19, CD22, or Mock T cells, is mediated successfully through either the CD19 scFv or CD22 scFv ( $n=1$ donor, technical triplicates) b. Mean fluorescence intensity measured via a fluorescently tagged CD22 molecule.c, CAR transduction efficiency and vector copy number. For b-c, $n=5$ CD22-BB.z products, 11 CD19-22.BB.z. 


\section{Reporting Summary}

Nature Portfolio wishes to improve the reproducibility of the work that we publish. This form provides structure for consistency and transparency in reporting. For further information on Nature Portfolio policies, see our Editorial Policies and the Editorial Policy Checklist.

\section{Statistics}

For all statistical analyses, confirm that the following items are present in the figure legend, table legend, main text, or Methods section.

$\mathrm{n} / \mathrm{a} \mid$ Confirmed

$\bigotimes$ The exact sample size $(n)$ for each experimental group/condition, given as a discrete number and unit of measurement

$\square$ \ A statement on whether measurements were taken from distinct samples or whether the same sample was measured repeatedly

The statistical test(s) used AND whether they are one- or two-sided

Only common tests should be described solely by name; describe more complex techniques in the Methods section.

Х $\square$ A description of all covariates tested

\A description of any assumptions or corrections, such as tests of normality and adjustment for multiple comparisons

A full description of the statistical parameters including central tendency (e.g. means) or other basic estimates (e.g. regression coefficient)

AND variation (e.g. standard deviation) or associated estimates of uncertainty (e.g. confidence intervals)

For null hypothesis testing, the test statistic (e.g. $F, t, r$ ) with confidence intervals, effect sizes, degrees of freedom and $P$ value noted

Give $P$ values as exact values whenever suitable.

Х $\square$ For Bayesian analysis, information on the choice of priors and Markov chain Monte Carlo settings

Х $\square$ For hierarchical and complex designs, identification of the appropriate level for tests and full reporting of outcomes

\ $\square$ Estimates of effect sizes (e.g. Cohen's $d$, Pearson's $r$ ), indicating how they were calculated

Our web collection on statistics for biologists contains articles on many of the points above.

\section{Software and code}

Policy information about availability of computer code

Data collection BCFACs Diva and BD LSRII for flow cytometry

Data analysis Statistics: R version 3.6.2 or GraphPad Prism 8, Flow cytometry FloJo v 8 and CytoBank. Isopeak 2.8.0.0 was used for single cell cytokine analysis.R code was written to perform basic analyses and do not entail a custom code or algorithm.

For manuscripts utilizing custom algorithms or software that are central to the research but not yet described in published literature, software must be made available to editors and

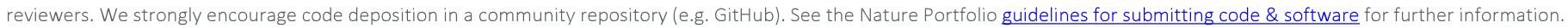

\section{Data}

Policy information about availability of data

All manuscripts must include a data availability statement. This statement should provide the following information, where applicable:

- Accession codes, unique identifiers, or web links for publicly available datasets

- A description of any restrictions on data availability

- For clinical datasets or third party data, please ensure that the statement adheres to our policy

All requests for raw and analyzed data will be reviewed by the corresponding author to determine if the request if subject to any intellectual property of confidentiality considerations. Patient-related data not included in the paper were generated as part of clinical trials and may be subject to patient confidentiality. Any data and materials that can be shared will be released via a Material Transfer Agreement. Source data for Figures 1-4 and Extended Figures 1-7 are provided with the manuscript. CD19-22 Bispecific CAR sequence is in the patent application: U.S. Provisional Patent Application No. 62/135,442 filed March 19, 2015, and which is entitled "DUAL SPECIFIC ANTI-CD22-ANTI-CD19CHIMERIC ANTIGEN RECEPTORS" and the amino acid sequence is provided in Supplementary Figure 2. 
Please select the one below that is the best fit for your research. If you are not sure, read the appropriate sections before making your selection.

$\bigotimes$ Life sciences $\quad \square$ Behavioural \& social sciences $\quad \square$ Ecological, evolutionary \& environmental sciences

For a reference copy of the document with all sections, see nature.com/documents/nr-reporting-summary-flat.pdf

\section{Life sciences study design}

All studies must disclose on these points even when the disclosure is negative.

Sample size All infused with CD19-22.BB.z patients were included in analysis of clinical outcomes. Axi-cel patients with available IHC and/or quantitative flow cytometry were included for analysis. No formal sample size calculation was made.

Data exclusions No data were excluded

Replication All experiments reported in the paper were from patient samples. Data from Extended Figure 7A was performed with samples from a healthy donor and run successfully in technical triplicate.

Randomization This was a Phase I to test safety and tolerability and therefore randomization was not performed. Standard of care axi-cel patients were not randomized.

Blinding No blinding was performed in this Phase I trial.

\section{Reporting for specific materials, systems and methods}

We require information from authors about some types of materials, experimental systems and methods used in many studies. Here, indicate whether each material, system or method listed is relevant to your study. If you are not sure if a list item applies to your research, read the appropriate section before selecting a response.

\begin{tabular}{|c|c|}
\hline $\mathrm{n} / \mathrm{a}$ & Involved in the study \\
\hline & \ Antibodies \\
\hline & $\bigotimes$ Eukaryotic cell lines \\
\hline Х & $\square$ Palaeontology and archaeology \\
\hline Х & $\square$ Animals and other organisms \\
\hline L & $\bigotimes$ Human research participants \\
\hline & $\bigotimes$ Clinical data \\
\hline Х & $\square$ Dual use research of concern \\
\hline
\end{tabular}

Methods

$\mathrm{n} / \mathrm{a}$ Involved in the study

X $\square$ ChIP-seq

$\square$ \ Flow cytometry

X| MRI-based neuroimaging

\section{Antibodies}

Antibodies used

All antibodies used, manufacturer, part number, respective fluorochromes and dilutions are listed in the methods section in: Panel 1, Antibody Index for Phenotyping and Exhaustion Profiling, Panel 2, and Panel 3.

Validation

All commercially-available antibodies are validated and routinely tested as described in the manufacturers product information (BD
Biosciences, Biolegend, Miltenyi and Thermo Fisher). The one non-commerically available antibody is CD19 anti-ld-DL650/APC which
was validated for specificity in Jena et. al, PLOSOne 2013., We have conjugated, validated, and titrated in our lab to determine the
appropriate volume to use for staining. We also conduct stability testing on this reagent to ensure the quality. We compared
antibody-specific staining to isotype, FMO, or no staining control samples and we use the same lot numbers of antibody when
possible in our panels. Primary antibodies have been titrated to determine the optimal stain concentration for each test. This data is
included in Supplemental Figure 3.

\section{Eukaryotic cell lines}

Policy information about cell lines

Cell line source(s)

NALM6 from NCI (Terry Fry) - lines were CRISPR/gene edited to create NALM6 CD22KO, NALM6 CD19KO, and NALM6 DO KO lines

Authentication

STR DNA profiling of all cell lines is conducted by Genetica Cell Line testing once per year

Mycoplasma contamination

All stock NALM6 vials were tested for mycoplasma and found to be negative. 


\section{Human research participants}

\section{Policy information about studies involving human research participants}

Population characteristics

Two disease cohorts were treated in this Phase I trial. Patients with aggressive large B-cell lymphoma relapsed or refractory after 2 lines of therapy including DLBCL, PMBCL, and large cell lymphoma transformed from follicular or other indolent Iymphomas were eligible. Patients with B-acute lymphoblastic leukemia relapsed or refractory to 2 lines of therapy were eligible. Patient characteristics are described in Results, Table 1 A and B and Methods. Standard of care axi-cel patients consisted of patients with aggressive large cell lymphoma relapsed or refractory after 2 lines of therapy.

Recruitment

Patients were not actively recruited and were enrolled on a first-come first-serve basis. The trial patients reflect our overall patient composition both in ethnicity and age range at our academic medical center.

Ethics oversight

The Phase I study as well as the Clinical Bio-repository was governed by the Institutional Review Board of the Stanford Cancer Center

Note that full information on the approval of the study protocol must also be provided in the manuscript.

\section{Clinical data}

Policy information about clinical studies

All manuscripts should comply with the ICMJE guidelines for publication of clinical research and a completed CONSORT checklist must be included with all submissions.

Clinical trial registration

Study protocol

Data collection

Outcomes

\section{Clinicaltrials.gov NCT03233854}

The full protocol is included in the Supplementary Materials

All data was collected between September 11,2017 and June 15, 2020 at Stanford Cancer Center

In the Methods we included the following: Primary outcomes were feasibility of manufacture of CD19-22.BB.Z-CAR as well as safety of CD19-22.BB.Z-CAR. Secondary outcomes included efficacy of CD19-22.BB.Z-CAR to induce clinical response at 3 months for LBCL and 1 month for B-ALL. Exploratory objectives included the rate of CD19 negative relapse after CD19-22.BB.Z-CAR.

\section{Flow Cytometry}

Plots

Confirm that:

$\bigotimes$ The axis labels state the marker and fluorochrome used (e.g. CD4-FITC).

\The axis scales are clearly visible. Include numbers along axes only for bottom left plot of group (a 'group' is an analysis of identical markers).

\All plots are contour plots with outliers or pseudocolor plots.

\A numerical value for number of cells or percentage (with statistics) is provided.

\section{Methodology}

Sample preparation

Instrument

Software

Cell population abundance

Gating strategy

Debris was gated out and samples were gated on FSC/SSC lymphocyte gate, on singlets using FSC-A/FSC-H, viable cells or CD3 cells as applicable for the figure. Beads were used for compensation and FMO controls were used in multi-color panels to assist with gating strategies. In some instances, healthy donor CART were run in parallel as a control and to check batch normalization. 